\title{
FLUX AND SCATTERING INTO CONES FOR LONG RANGE AND SINGULAR POTENTIALS
}

\author{
W.O. Amrein \\ Department of Theoretical Physics \\ University of Geneva \\ CH-1211 Geneva 4 \\ D.B. Pearson * \\ Department of Applied Mathematics \\ University of Hull \\ Hull HU6 7RX, United Kingdom
}

\begin{abstract}
We prove that the probability of finding a scattered quantum-mechanical particle at large times in a truncated cone is identical with the scattered flux, integrated over time, across a distant spherical surface subtending this cone. The theory applies to potentials with arbitrary local singularities and decaying faster than $r^{-2 / 3}$ at large distances.
\end{abstract}

CERN-TH/96-370

December 1996

* Partially supported by the Swiss National Science Foundation 


\section{Introduction}

The definition of scattering cross sections in the Hilbert space formulation of quantum scattering theory is based on Dollard's scattering into cones formula (see e.g. [1],[2]). This formula expresses the probability of finding the scattered particles in the far future in a given cone in terms of the scattering operator S. As mentioned in [3], the relevance of such arguments relies on the assumption that that the probability of finding a scattered particle at large times in some cone is identical with the scattered flux, integrated over time, across a distant surface subtending this cone. The validity of this assumption has recently been established for free particles and for scattering by short range potentials, see [4]-[6]. The method of estimating the scattered flux applied in [6] does not however cover Coulomb potentials, and it is the purpose of the present paper to introduce a different approach which allows one to treat also Coulomb and other long range potentials in two-body scattering. Moreover the theory will cover potentials with strong local singularities. The extension of the results to $\mathrm{N}$-body systems will be communicated elsewhere.

In Section 2 we introduce the mathematical notions needed to describe the flux across certain 2-dimensional surfaces embedded in $\mathbb{R}^{3}$. The divergence theorem can be used to relate the flux across the boundary of a (reasonably shaped) truncated cone to the probability of presence in that cone (Section 3); if the flux across the lateral surface of the cone is sufficiently small at large times, one can then deduce the validity of the assumption mentioned above. Details, together with precise general conditions on the flux, will be given in Section 4 . In Section 5 we use propagation estimates to prove that these conditions on the flux are satisfied for hamiltonians with smooth potentials (including certain long range potentials), and in Section 6 this result is extended to potentials with local singularities, by the use of relative wave operators.

We employ the following notations: $\mathbf{Q}=\left(\mathrm{Q}_{1}, \mathrm{Q}_{2}, \mathrm{Q}_{3}\right)$ and $\mathbf{P}=\left(\mathrm{P}_{1}, \mathrm{P}_{2}, \mathrm{P}_{3}\right)$ denote the usual 3-component position and momentum operator respectively in the Hilbert space $\mathcal{H}=$ $\mathrm{L}^{2}\left(\mathbb{R}^{3}\right)$, with $\hbar=1$. We set $\mathrm{Q}=\left\{\Sigma_{\mathrm{j}=1}^{3} \mathrm{Q}_{\mathrm{j}}^{2}\right\}^{1 / 2},<\mathrm{Q}>\rho=\left(\mathrm{I}+\mathrm{Q}^{2}\right)^{\rho / 2}$ for $\rho \in \mathbb{R}, \mathrm{H}_{\mathrm{o}}=\mathbf{P}^{2}=$ $\Sigma_{j=1}^{3} P_{j}^{2}$ and $P=H_{o}^{1 / 2}$. Each of these operators is considered on the domain on which it is self-adjoint. If $\varphi$ is a function defined on $\mathbb{R}^{3}$, then $\varphi(\mathbf{Q})$ is the operator of multiplication by $\varphi(x)$ in $L^{2}\left(\mathbb{R}^{3}\right)$. In the particular case where $\varphi$ is the characteristic function $\chi_{G}$ of a subset $G$ of $\mathbb{R}^{3}\left(\chi_{G}(\mathbf{x})=1\right.$ if $\mathbf{x} \in G, \chi_{G}(\mathbf{x})=0$ if $\left.\mathbf{x} \in G\right)$, we use the notation $E(x \in G)$ for $\chi_{G}(\mathbf{Q})$. Sometimes we write simply $\varphi$ f for $\varphi(\mathbf{Q})$ f. By $\|\cdot\|$ we mean the norm and by $\langle\cdot, \cdot\rangle$ the scalar product in $L^{2}\left(\mathbb{R}^{3}\right.$ ), whereas the $L^{2}$-norm and scalar product on a subset $G$ of $\mathbb{R}^{3}$ (always with respect to Lebesgue measure) will be written as $\|\cdot\|_{L^{2}(G)}$ and $\langle\cdot, \cdot\rangle_{L^{2}(G)}$ respectively. We observe that $D(P)=\cap_{j=1}^{3} D\left(P_{j}\right)$ and $\|P f\|^{2}=\Sigma_{j=1}^{3}\left\|P_{j} f\right\|^{2}$ if $f$ belongs to the domain $D(P)$ of $P$. We denote by $\boldsymbol{B}(\mathcal{H})$ the set of all bounded everywhere defined linear operators in $\mathcal{H}$ and by $\|A\|$ the norm of $A \in B(\mathcal{H})$. If $\alpha=\left(\alpha_{1}, \alpha_{2}, \alpha_{3}\right)$ is a multi-index, then $|\alpha|=\alpha_{1}+\alpha_{2}+\alpha_{3}$ and

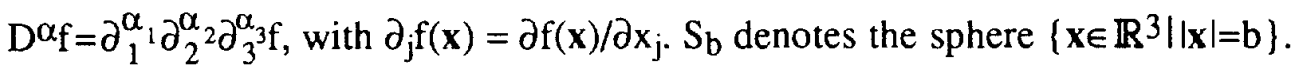




\section{Restriction of Functions to the Boundaries of Cones}

Throughout this paper $C$ will be a closed cone in $\mathbb{R}^{3}$ with vertex at the origin and different from $\mathbb{R}^{3}\left(C \neq \mathbb{R}^{3}\right)$. We let a be some fixed positive number $(a>0)$. If $R \in(a, \infty)$, we denote by $C_{a, R}$ the doubly truncated cone $C_{a, R}=\{x \in C l a<|x|<R\}$. The boundary $\partial C_{a, R}$ of $C_{a, R}$ is the union of two spherical surfaces $\Sigma_{a}=\{x \in C|| x \mid=a\}$ and $\Sigma_{R}=\{x \in C|| x \mid=R\}$ and the lateral surface $\Lambda_{a, R}=\{x \in \partial C l a<|x|<R\}$. We shall also consider the once truncated cone $\mathrm{C}_{\mathrm{a}} \equiv \mathrm{C}_{\mathrm{a}, \infty}=\{\mathbf{x} \in \mathrm{C}|| \mathbf{x} \mid>\mathrm{a}\}$, with corresponding lateral surface $\Lambda_{\mathrm{a}}=\{\mathbf{x} \in \partial \mathrm{C}|| \mathbf{x} \mid>\mathrm{a}\}$.

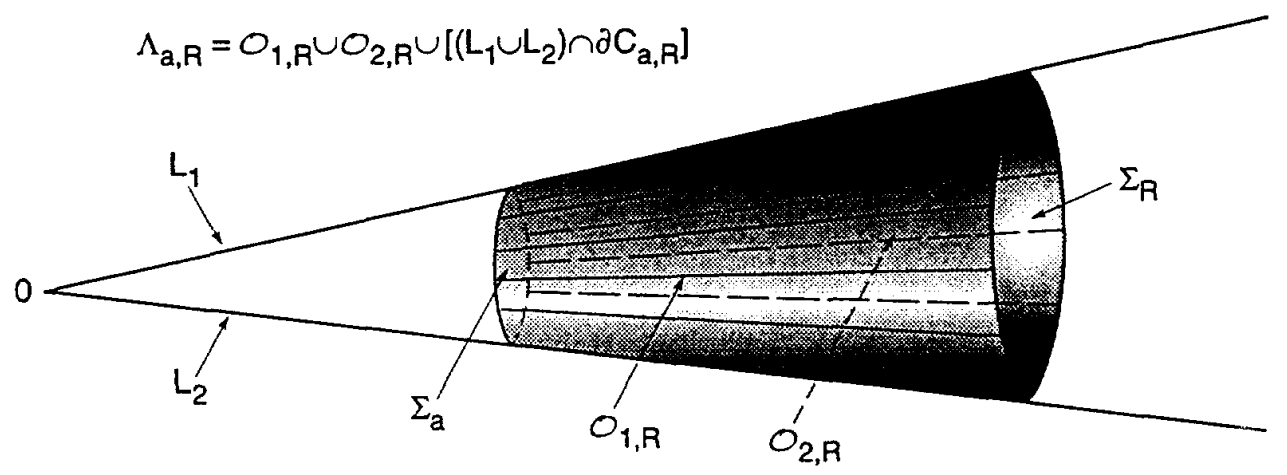

Figure 1. A doubly truncated admissible cone

To prove our results we shall restrict ourselves to specially simple cones which we call admissible. The extension to a much larger class of cones is then straightforward and will be discussed at the end of Section 4 .

A closed cone $C \neq \mathbb{R}^{3}$ will be called admissible if it is convex, with non-empty interior, and if there are a finite number of straight lines $\left\{L_{k}\right\}_{k=1}^{N}$ on its boundary $\partial C$, each passing through the point $\mathrm{x}=0$, that subdivide $\Lambda_{\mathrm{a}}$ into $\mathrm{N}$ disjoint smooth surfaces $\Theta_{\mathrm{k}}$. So we have $\Lambda_{\mathrm{a}}$ $=\left(U_{k=1}^{N} \Theta_{k}\right) \cup\left(U_{k=1}^{N} L_{k} \cap \partial C_{a}\right)$, where it is assumed that $L_{k} \cap \Theta_{j}=\varnothing$ for all j,k (i.e. $\Theta_{k}$ does not contain its boundary points on $\Lambda_{\mathrm{a}}$ ).

If $\mathrm{C}$ is an admissible cone and $\mathrm{R}<\infty$, one can apply the divergence theorem on the domain $C_{a, R}$ (see e.g. [7],[8]): if $u, v$ are functions of class $C^{2}$ on $\mathbb{R}^{3}$ and $\mathcal{O}_{k, R}=\left\{x \in \mathcal{O}_{k}|| x \mid\right.$ $<\mathrm{R}\}$, then

$$
\begin{aligned}
\int_{C_{a, R}}[u(x) \Delta v(x)-v(x) \Delta u(x)] d^{3} x= & \Sigma_{k=1}^{N} \int_{\Theta_{k}}[u(y) \nabla v(y)-v(y) \nabla u(y)] \cdot n(y) d \sigma(y) \\
& +\int_{\Sigma_{a} u \Sigma_{R}}[u(y) \nabla v(y)-v(y) \nabla u(y)] \cdot n(y) d \sigma(y),
\end{aligned}
$$


where d $\sigma$ denotes the area element on the surface under consideration and $\mathbf{n}(\mathbf{y})$ is the outward unit normal vector to this surface at the point $y$.

Let $\mathrm{C}$ be an admissible cone and let $\theta$ be either one of the $\theta_{\mathrm{k}}$ or $\theta=\Sigma_{\mathrm{b}}=\mathrm{C}_{\mathrm{S}} \mathrm{b}$ for some $b>0$. If $f$ is a smooth function on $\mathbb{R}^{3}$, then its restriction $f l_{\odot}$ to $\theta$ is well defined. We shall need estimates on the norm of $f l_{\Theta}$ in $L^{2}(\theta)$ in terms of certain norms on $f$ in $L^{2}\left(\mathbb{R}^{3}\right)$. More precisely, we have the following inequality the proof of which is indicated in the Appendix:

Proposition 1 : If $\theta$ is as above, then there is a constant $c<\infty$ such that the following inequality holds for all functions $f, \varphi: \mathbb{R}^{3} \rightarrow \mathbb{C}$ of class $C^{1}$ such that (i) $f$ belongs to the domain $D(P)$ of the operator $P=H_{o}^{1 / 2}$ (in particular $f \in L^{2}\left(\mathbb{R}^{3}\right)$ ) and (ii) $\varphi$ and its first order derivatives are bounded and $\varphi(\mathbf{x})=1$ for all $\mathbf{x} \in \mathcal{O}$ :

$$
\left\|\left.f\right|_{\Theta}\right\|_{L^{2}(\Theta)} \equiv\left[\int_{\Theta}|f(y)|^{2} d \sigma(y)\right]^{1 / 2} \leq c[\|\varphi(Q) f\|+\|P \varphi(Q) f\|]
$$

For $\varphi \equiv 1$, the inequality (2) means that the operator $\gamma_{\Theta}$ of restricting to $\theta$, defined first on the dense set of vectors $f$ indicated in Proposition $1\left(\gamma_{\Theta} f=\left.f\right|_{\Theta}\right)$, extends to a bounded operator from $\mathcal{H}^{1}\left(\mathbb{R}^{3}\right) \equiv \mathrm{D}(\mathrm{P})$ (equipped with the graph norm) to $\mathrm{L}^{2}(\Theta)$, or equivalently that the closure $\Gamma_{\Theta}$ of $\gamma_{\Theta}(I+P)^{-1}$ is a bounded operator from $L^{2}\left(\mathbb{R}^{3}\right)$ to $L^{2}(\theta)$; in fact, upon replacing $f$ by $(I+P)^{-1} g$ in (2) and observing that $\|f\|+\|P f\| \leq \sqrt{2}\|(I+P) f\|$ (since $P \geq 0$ ), one sees that $\left\|\Gamma_{\Theta} g\right\|_{L^{2}(\Theta)} \leq \sqrt{2} \mathrm{c}\|g\|$. The adjoint $\Gamma_{\Theta}^{*}$ of $\Gamma_{\Theta}$ is then a bounded operator from $L^{2}(\theta)$ to $\mathrm{L}^{2}\left(\mathbb{R}^{3}\right)$.

We are particularly interested in the operator $\gamma_{\Lambda_{\mathrm{a}}}$ of restricting functions to the lateral boundary $\Lambda_{\mathrm{a}}$ of $\mathrm{C}_{\mathrm{a}}: \gamma_{\Lambda_{\mathrm{a}}} \mathrm{f}=\left.\mathrm{f}\right|_{\Lambda_{\mathrm{a}}}$. It is clear that $\left\|\left.f\right|_{\Lambda_{\mathrm{a}}}\right\|_{L^{2}\left(\Lambda_{\mathrm{a}}\right)}^{2}=\Sigma_{\mathrm{k}=1}^{\mathrm{N}}\left\|\left.f\right|_{\Theta_{\mathrm{k}}}\right\|_{L^{2}\left(\Theta_{\mathrm{k}}\right)}^{2}$. If $L^{2}\left(\Theta_{k}\right)$ is identified in a natural way with a subspace of $L^{2}\left(\Lambda_{a}\right)$, then $\Gamma_{\theta_{k}}$ becomes a bounded operator from $L^{2}\left(\mathbb{R}^{3}\right)$ into $L^{2}\left(\Lambda_{a}\right)$, and the closure $\Gamma_{\Lambda_{a}}$ of $\gamma_{\Lambda_{a}}(I+P)^{-1}$ is simply given by

$$
\Gamma_{\Lambda_{\mathrm{a}}}=\Sigma_{\mathrm{k}=1}^{\mathrm{N}} \Gamma_{\vartheta_{\mathrm{k}}}
$$

Similarly, if $a<R<\infty$, one may view $L^{2}\left(\Lambda_{a, R}\right)$ as a subspace of $L^{2}\left(\Lambda_{a}\right)$ and introduce a bounded operator $\Gamma_{\Lambda_{\mathrm{a}, \mathrm{R}}}$ from $L^{2}\left(\mathbb{R}^{3}\right)$ into $L^{2}\left(\Lambda_{\mathrm{a}}\right)$ as the closure of $\gamma_{\Lambda_{\mathrm{a}, \mathrm{R}}}(\mathrm{I}+\mathrm{P})^{-1}$, with $\gamma_{\Lambda_{\mathrm{a}, \mathrm{R}}} f=$ $\left.f\right|_{\Lambda_{a}, R^{R}}$. The following relations are straightforward consequences of Proposition 1:

$$
\left\|\Gamma_{\Lambda_{\mathrm{a}, \mathrm{R}}}\right\| \leq\left\|\Gamma_{\Lambda_{\mathrm{a}}}\right\|<\infty \quad \forall \mathrm{R}>\mathrm{a} \quad, \quad \underset{\mathrm{R} \rightarrow \infty}{\mathrm{s}-\lim } \Gamma_{\Lambda_{\mathrm{a}, \mathrm{R}}}=\Gamma_{\Lambda_{\mathrm{a}}}
$$


Another useful result is the following estimate: let $\Gamma_{\Sigma_{b}}$ be the closure of $\gamma_{\Sigma_{b}}(I+P)^{-1}$, where $\gamma_{\Sigma_{b}} f=\left.f\right|_{\Sigma_{b}}$. Then, as shown in the Appendix, one has

$$
\left\|\Gamma_{\Sigma_{b}}\right\| \leq 4 \quad \text { for all } b \geq 1
$$

(Observe that this implies the estimate (2.2) in [6]; the operator denoted by $\Gamma_{R}$ in [6] may be identified with $R^{(n-1) / 2} \Gamma_{\Sigma_{R}}$.

\section{The Quantum-Mechanical Flux}

Let $\mathcal{Q}$ be a smooth hypersurface in $\mathbb{R}^{3}$ as in Proposition 1. If $\mathrm{f}$ is a wave function, then texts on quantum mechanics define the flux density of $f$ at a point $x \in \mathbb{R}^{3}$ as $2 \operatorname{Im} \overline{f(x)} \nabla f(x) \equiv$ $2 \operatorname{Re} \overline{f(x)} \operatorname{Pf}(x)$ (we choose the mass $m=1 / 2$ ), and the flux $\Phi_{\Theta}(f)$ through $\Theta$ is given as the integral of the normal component of this density over $\theta$ :

$$
\Phi_{\mathcal{O}}(f)=2 \operatorname{Re} \Sigma_{j=1}^{3} \int_{\mathcal{O}} \overline{f(y)} n_{j}(y)\left(P_{j} f\right)(y) d \sigma(y) .
$$

If $\mathrm{f}$ and $\nabla \mathrm{f}$ are continuous functions, the flux density has a pointwise meaning. For more general wave functions in $L^{2}\left(\mathbb{R}^{3}\right)$, the integral in (6) need not be well defined, because $\theta$ is a set of Lebesgue measure zero in $\mathbb{R}^{3}$, so that the integrand may not make sense. However, for $f \in D\left(H_{0}\right)$, one may give a meaning to the integrated flux through $\Theta$ as the expectation value of a certain operator $\hat{F}_{\mathcal{O}}$, the so-called flux operator for $\theta$, defined in terms of $\Gamma_{\mathcal{O}}$. To see this, consider $\mathbf{n}(\mathbf{y})=\left(\mathrm{n}_{1}(\mathbf{y}), \mathrm{n}_{2}(\mathbf{y}), \mathrm{n}_{3}(\mathbf{y})\right)$ as a multiplication operator in $\mathrm{L}^{2}(\theta)$ and rewrite (6) (for smooth $\mathrm{f}$ in the first instance) as

$$
\begin{aligned}
\Phi_{\Theta}(f) & =2 \operatorname{Re} \Sigma_{j=1}^{3} \int_{\Theta} \overline{\left(\gamma_{\Theta} f\right)(y)} n_{j}(y)\left(\gamma_{\Theta} P_{j} f\right)(y) d \sigma(y)=2 \operatorname{Re} \Sigma_{j=1}^{3}\left\langle\gamma_{\Theta} f, n_{j} \gamma_{\Theta} P_{j} f\right\rangle_{L} 2(\Theta) \\
& =2 \operatorname{Re} \Sigma_{j=1}^{3}\left\langle\gamma_{\Theta}(I+P)^{-1}(I+P) f, n_{j} \gamma_{\Theta}(I+P)^{-1} P_{j}(I+P) f\right\rangle_{L}(\Theta) \\
& =2 \operatorname{Re} \Sigma_{j=1}^{3}<(I+P) f, \Gamma_{\Theta}^{*} n_{j} \Gamma_{\Theta} P_{j}(I+P) f>=\operatorname{Re}<(I+P) f, F_{\Theta}\left(I+H_{0}\right) f>,
\end{aligned}
$$

where $F_{\odot}$ is the following bounded operator in $\mathcal{H}=\mathrm{L}^{2}\left(\mathbb{R}^{3}\right)$ :

$$
F_{\mathcal{Q}}=2 \Sigma_{j=1}^{3} \Gamma_{\mathcal{O}}^{*} n_{j} \Gamma_{\mathcal{Q}} P_{j}(I+P)\left(I+P^{2}\right)^{-1}
$$

Observe that (7), defined originally for smooth $f$, makes sense for all $f$ in $D\left(H_{0}\right)$. 
Eq. (7) shows that $\Phi_{\odot}(\mathrm{f})$ may be viewed as the expectation value $\left\langle\mathrm{f}, \hat{\mathrm{F}}_{\odot} \mathrm{f}\right\rangle$ of the (unbounded) observable $\hat{F}_{\Theta}=2 \operatorname{Re} \Sigma_{j=1}^{3}(I+P) \Gamma_{\Theta}^{*} n_{j} \Gamma_{\Theta} P_{j}(I+P)$. For our purposes, however, it will be more convenient to use the last expression in (7) for $\Phi_{\Theta}(f)$ in terms of the non-selfadjoint, but bounded, operator $F_{\odot}$.

We draw attention to a useful alternative expression for $\Phi_{\mathcal{O}}(f)$. If $\Theta$ is any subset of $\mathbb{R}^{3}$, define $\Theta_{\mathcal{O}}$ to be the set of all functions $\theta: \mathbb{R}^{3} \rightarrow \mathbb{C}$ of class $C^{2}$ such that $\theta, \partial_{j} \theta$ and $\Delta \theta$ are bounded and such that $\theta(\mathbf{x})=1$ for all $\mathbf{x}$ in some neighbourhood of $\Theta$. If $\Theta$ is as in (7), and if $\theta_{1}, \theta_{2} \in \Theta_{\mathcal{O}}$, then the first expression for $\Phi_{\Theta}(f)$ in (7) remains unchanged if $\left(\gamma_{\Theta} f\right)(y)$ is replaced by $\left(\gamma_{\Theta} \theta_{1} f\right)(y)$ and $\left(\gamma_{\Theta} P_{j} f\right)(y)$ by $\left(\gamma_{\Theta} P_{j} \theta_{2} f\right)(y)$. So, for $\theta_{1}, \theta_{2} \in \Theta_{\Theta}$, we have

$$
\Phi_{\Theta}(\mathrm{f})=\operatorname{Re}<(\mathrm{I}+\mathrm{P}) \theta_{1}(\mathbf{Q}) \mathrm{f}, \mathrm{F}_{\odot}\left(\mathrm{I}+\mathrm{H}_{\mathrm{o}}\right) \theta_{2}(\mathbf{Q}) \mathrm{f}>
$$

Now consider a doubly truncated admissible cone $C_{a, R}$. The boundary of $C_{a, R}$ is the union of a finite number of smooth hypersurfaces, namely $\Sigma_{a}, \Sigma_{R}$ and $\Theta_{k, R}=\Theta_{k} \cap \partial C_{a, R}$ $(k=1, \ldots, N)$ and of a finite number of curves, so one may associate to it $i$ flux operator $F_{\partial C_{a, R}}$ by setting

$$
F_{\partial C_{a, R}}=F_{\Sigma_{a}}+F_{\Sigma_{R}}+\Sigma_{k=1}^{N} F_{\Theta_{k, R}}
$$

Let $\mathrm{H}$ be a hamiltonian of the form $\mathrm{H}=\mathrm{H}_{0}+\mathrm{V}(\mathrm{Q})$ and $\mathrm{U}_{\mathrm{l}}=\exp (-\mathrm{iHt})$ the associated unitary evolution operator for a time interval of length $t$ (recall that we set $\hbar=1$ ). We consider for the moment potentials $V$ such that $D\left(H_{0}\right) \subseteq D(V)$ and such that $H=H_{0}+V(Q)$ is self-adjoint on $\mathrm{D}\left(\mathrm{H}_{\mathrm{o}}\right)$, so that $\mathrm{D}(\mathrm{H})=\mathrm{D}\left(\mathrm{H}_{\mathrm{O}}\right)$ (this holds in particular if $\mathrm{V}=\mathrm{V}_{1}+\mathrm{V}_{2}$ with $\mathrm{V}_{1}$ a bounded function and $V_{2} \in L^{2}\left(\mathbb{R}^{3}\right)$; more singular potentials will be considered in Section 6). One expects that the rate of change of the probability of presence of a state in $C_{a, R}$ should be determined by the flux through the boundary $\partial \mathrm{C}_{\mathrm{a}, \mathrm{R}}$ of the truncated cone $\mathrm{C}_{\mathrm{a}, \mathrm{R}}$. To make this precise, let us observe that, if $g \in D(H)$, then $U_{t} g \in D(H)$ and consequently

$$
\begin{aligned}
\frac{d}{d t}\left\|E\left(x \in C_{a, R}\right) U_{t} g\right\|^{2} & =\frac{d}{d t}<U_{t} g, E\left(x \in C_{a, R}\right) U_{t} g> \\
& =i\left[<H_{t} g, E\left(x \in C_{a, R}\right) U_{t} g>-<U_{t} g, E\left(x \in C_{a, R}\right) H U_{t} g>\right] \\
& =i\left[<H_{o} U_{t} g, E\left(x \in C_{a, R}\right) U_{t} g>-<U_{t} g, E\left(x \in C_{a, R}\right) H_{o} U_{t} g>\right],
\end{aligned}
$$

since $\mathrm{V}(\mathbf{Q}) \mathrm{E}\left(\mathbf{x} \in \mathrm{C}_{\mathrm{a}, \mathrm{R}}\right)=\mathrm{E}\left(\mathbf{x} \in \mathrm{C}_{\mathrm{a}, \mathrm{R}}\right) \mathrm{V}(\mathbf{Q})$. With $\mathrm{H}_{\mathbf{0}}=-\Delta$, formal application of the divergence theorem (1) suggests that

$$
\frac{\mathrm{d}}{\mathrm{dt}}\left\|\mathrm{E}\left(\mathbf{x} \in \mathrm{C}_{\mathrm{a}, \mathrm{R}}\right) \mathrm{U}_{\mathrm{t}} \mathrm{g}\right\|^{2}=-\Phi_{\partial \mathrm{C}_{\mathrm{a}, \mathrm{R}}}\left(\mathrm{U}_{\mathrm{t}} \mathrm{g}\right)
$$

The following lemma, applied with $h=U_{t} g$ to the identity (11), will allow us to verify rigorously that (11) implies (12). 
Lemma 1 : If $h \in D\left(H_{0}\right)$, then

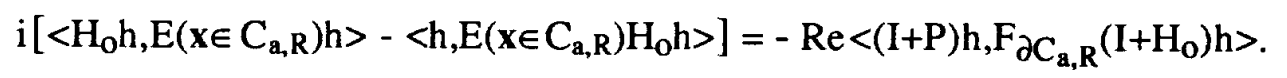

Proof : To simplify notation we write $E$ for $E\left(x \in C_{a, R}\right)$. Choose $\varphi \in C_{0}^{\infty}\left(\mathbb{R}^{3}\right)$ such that

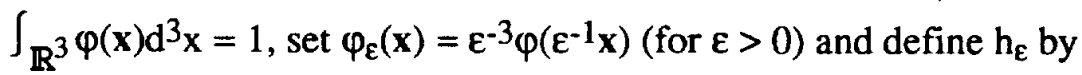

$$
h_{\varepsilon}(\mathbf{x})=\int_{\mathbb{R}^{3}} \varphi_{\varepsilon}(\mathbf{x}-\mathbf{y}) \mathrm{h}(\mathbf{y}) \mathrm{d}^{3} \mathrm{y} \equiv\left(\varphi_{\varepsilon} * h\right)(\mathbf{x})
$$

Then $h_{\varepsilon}$ is of class $C^{\infty}$ and $\left\|h_{\varepsilon}-h\right\| \rightarrow 0$ as $\varepsilon \rightarrow 0$ (see e.g. Lemma 2.18 of [9]). Also, since $h \in D\left(H_{0}\right)$ and $D^{\alpha} h_{\varepsilon}=\varphi_{\varepsilon} * D^{\alpha} h$, we have $\left\|P_{j} h_{\varepsilon}-P_{j} h\right\| \rightarrow 0$ and $\left\|P_{j} P_{k} h_{\varepsilon}-P_{j} P_{k} h\right\| \rightarrow 0$ as $\varepsilon \rightarrow 0$. So from the divergence theorem (1) and using (6), (7) and (10), we have

$$
\begin{aligned}
& <\mathrm{H}_{\mathrm{o}} \mathrm{h}, \mathrm{Eh}>-<\mathrm{h}, \mathrm{EH}_{\mathrm{O}} \mathrm{h}>=\lim _{\varepsilon \rightarrow 0}\left[<\mathrm{H}_{\mathrm{o}} \mathrm{h}_{\mathcal{E}}, \mathrm{Eh}_{\mathcal{\varepsilon}}>-<\mathrm{h}_{\mathcal{E}}, \mathrm{EH}_{\mathrm{o}} \mathrm{h}_{\mathcal{\varepsilon}}>\right] \\
& =\lim _{\varepsilon \rightarrow 0} \int_{C_{a, R}}\left[\overline{h_{\varepsilon}(\mathbf{x})} \Delta h_{\varepsilon}(\mathbf{x})-h_{\varepsilon}(\mathbf{x}) \overline{\Delta h_{\varepsilon}(\mathbf{x})}\right] d^{3} \mathbf{x} \\
& =\lim _{\varepsilon \rightarrow 0} \int_{\Sigma_{\mathrm{a}} \cup \Sigma_{\mathbf{R}} \cup \Lambda_{\mathrm{a}, \mathrm{R}}}\left[\overline{h_{\varepsilon}(\mathbf{y})} \nabla \mathrm{h}_{\boldsymbol{\varepsilon}}(\mathbf{y})-\mathrm{h}_{\boldsymbol{\varepsilon}}(\mathbf{y}) \overline{\nabla \mathrm{h}_{\boldsymbol{\varepsilon}}(\mathbf{y})}\right] \cdot \mathbf{n}(\mathbf{y}) \mathrm{d} \sigma(\mathbf{y}) \\
& =\mathrm{i} \lim _{\varepsilon \rightarrow 0} \operatorname{Re}<(\mathrm{I}+\mathrm{P}) \mathrm{h}_{\mathcal{\varepsilon}}, \mathrm{F}_{\partial \mathrm{C}_{\mathrm{a}, \mathrm{R}}}\left(\mathrm{I}+\mathrm{H}_{\mathrm{O}}\right) \mathrm{h}_{\mathcal{\varepsilon}}>=\mathrm{i} \operatorname{Re}<(\mathrm{I}+\mathrm{P}) \mathrm{h}, \mathrm{F}_{\partial \mathrm{C}_{\mathrm{a}, \mathrm{R}}}\left(\mathrm{I}+\mathrm{H}_{\mathrm{O}}\right) \mathrm{h}>
\end{aligned}
$$

Remark : As mentioned at the beginning of this section, a pointwise meaning can be found for the flux density in situations where $f$ and $\nabla f$ are continuous functions on $\mathbb{R}^{3}$. The condition that $f \in D\left(H_{0}\right)$, which is normally assumed here, implies that $f$ is continuous. The continuity of $\nabla f$ is assured for example by the assumption that $f \in D\left(H_{0}^{v}\right.$ ) for some $v>5 / 4$ (see for example Theorem IX.24 of [10]). In our applications we have $f=U_{t} g=U_{t} \Omega_{-} f_{i n}=\Omega_{-} U_{t}^{0} f_{i n}$, where $f_{\text {in }}$ is the initial wave function in a scattering process, $U_{t}^{o}=\exp \left(-\mathrm{iH}_{0} t\right)$ and $\Omega_{\text {- the wave }}$ operator at $t=-\infty$ (assumed to exist). If $f_{i n} \in D\left(H_{o}^{\rho}\right)$ for some $\rho>0$, then $U_{t}^{o} f_{i n} \in D\left(H_{0}^{\rho}\right)$ and $U_{t} \Omega_{-} f_{\text {in }} \in D\left(|H|^{\rho}\right)$. If $D(H)=D\left(H_{0}\right)$, then $f=U_{t} g$ is continuous for each value of $t$. The continuity of $\nabla U_{t}$ f follows from the assumption that $\Omega_{\text {. maps }} D\left(H_{o}^{\rho}\right)$, for some $\rho>0$, into $\mathrm{D}\left(\mathrm{H}_{\mathrm{O}}^{\mathrm{K}}\right)$, for some $\kappa>5 / 4$. Conditions on the potential $\mathrm{V}$ guaranteeing this mapping property of $\Omega$. can be found for example in [11]. 


\section{The Integrated Flux}

Let $\Theta$ again be a smooth hypersurface in $\mathbb{R}^{3}$ and $\left\{U_{t}\right\}_{t \in \mathbb{R}}$ a unitary evolution group. We denote by $I_{\mathcal{O}}(g, T)$ the time integral over the interval $(T, \infty)$ of the flux of $U_{t} g$ through $\theta$ :

$$
\mathrm{I}_{\mathcal{O}}(\mathrm{g}, \mathrm{T})=\int_{\mathrm{T}}^{\infty} \Phi_{\mathcal{O}}\left(\mathrm{U}_{\mathrm{t}} \mathrm{g}\right) \mathrm{dt}=\operatorname{Re} \int_{\mathrm{T}}^{\infty}<(\mathrm{I}+\mathrm{P}) \mathrm{U}_{\mathrm{L}} \mathrm{g}, \mathrm{F}_{\mathcal{Q}}\left(\mathrm{I}+\mathrm{H}_{\mathrm{O}}\right) \mathrm{U}_{\mathrm{t}} \mathrm{g}>\mathrm{dt}
$$

For a doubly truncated admissible cone $C_{a, R}$, this defines in particular $I_{\Sigma_{a}}(g, T), I_{\Sigma_{R}}(g, T)$ and the integrated flux $I_{\Lambda_{a, R}}(g, T)=\sum_{k=1}^{N} I_{\Theta_{k, R}}(g, T)$ through the lateral surface. We now list a set of conditions which imply that the probability of presence of $U_{t} g$ in $C_{a} \equiv C_{a, \infty}$ at time $t=+\infty$ is identical with the integrated flux $I_{\Sigma_{R}}(g, T)$ across $\Sigma_{R}$, in the limit $R \rightarrow \infty$ (for any finite $T$ ). These conditions are as follows:

$$
\begin{array}{ll}
\lim _{\mathrm{t} \rightarrow+\infty}\left\|E\left(x \in C_{a}, \mathrm{R}\right) U_{t} g\right\|=0 & \forall R \in(a, \infty) . \\
\lim _{R \rightarrow \infty} \int_{T_{1}}^{T_{2}} \Phi_{\Sigma_{R}}\left(U_{t} g\right) d t=0 & \text { if }-\infty<T_{1}<T_{2}<+\infty . \\
\lim _{T \rightarrow \infty} I_{\Sigma_{a}}(g, T) \equiv \lim _{T \rightarrow \infty} \int_{T}^{\infty} \Phi_{\Sigma_{a}}\left(U_{t} g\right) d t=0 .
\end{array}
$$

(H4) There are constants $R_{o}>a, t_{o}>0$ and a function $\eta \in L^{1}\left(t_{o}, \infty\right)$ such that $\left|\Phi_{\Lambda_{a, R}}\left(U_{t} g\right)\right| \leq \eta(t)$ for each $R \geq R_{o}$ and each $t \geq t_{0}$.

It is instructive to consider the interpretation of these conditions in terms of propagation properties of states. (H1) will hold if the state $U_{t} g$ is evanescent at large positive times, and (H2) requires that, if $\mathrm{R}$ is very large, then the flux through $\Sigma_{\mathrm{R}}$ be small at finite times. The condition (H3), as well as (H4) with $\mathrm{R}$ finite, demand a stronger form of evanescence: the flux of $U_{t} g$ through bounded hypersurfaces should be small at large times (think of $\eta$ for example as $\eta(t)=t^{-\mu}$ with $\mu>1$ ). A scattering state is expected to have all these properties. Since such states are asymptotically localized far away from the scattering center, it is the flux near infinity that matters at large times, and for large $\mathrm{R}$ the condition (H4) means that the flux should be essentially radial at large distances from the origin.

Proposition 2 : Let $\mathrm{C}$ be an admissible cone. Assume that $\mathrm{g} \in \mathrm{D}(\mathrm{H})$ is such that $(\mathrm{H} 1)-(\mathrm{H} 4)$ are satisfied and that equation (12) holds (in particular it is assumed that $\Phi_{\Sigma_{\mathrm{a}}}\left(\mathrm{U}_{\mathrm{t}} \mathrm{g}\right), \Phi_{\Sigma_{R}}\left(\mathrm{U}_{\mathrm{t}} \mathrm{g}\right)$ and $\Phi_{\Lambda_{a, R}}\left(U_{t} g\right)$ are well defined for each $t \in \mathbb{R}$ and each $\left.R>a\right)$. Then the following limits exist, are finite and equal for any finite $\mathrm{T}$ :

$$
\lim _{t \rightarrow+\infty}\left\|E\left(x \in C_{a}\right) U_{t} g\right\|^{2}=\lim _{R \rightarrow \infty} \int_{T}^{\infty} \Phi_{\Sigma_{R}}\left(U_{t} g\right) d t \equiv \lim _{R \rightarrow \infty} I_{\Sigma_{R}}(g, T)
$$

If in addition $\left\|\mathrm{E}(|\mathbf{x}| \leq \mathrm{a}) \mathrm{U}_{\mathrm{t}} \mathrm{g}\right\| \rightarrow 0$ as $\mathrm{t} \rightarrow+\infty$, then the limits in (16) are equal to $\lim _{t \rightarrow+\infty}\left\|E(x \in C) U_{t} g\right\|^{2}$. 
Proof : (i) We first deduce some consequences of (H4). From (8) and the second relation in (4), one sees that $F_{\Lambda_{a}, R}$ converges weakly to $F_{\Lambda_{a}}$ as $R \rightarrow \infty$. So $\lim _{R \rightarrow \infty} \Phi_{\Lambda_{a, R}}\left(U_{t} g\right)=$ $\Phi_{\Lambda_{\mathrm{a}}}\left(\mathrm{U}_{\mathrm{t}} \mathrm{g}\right)$, by virtue of (7) or (9). Then, by using (H4) and the Lebesgue dominated convergence theorem, we have for $t>t_{0}$ :

$$
\lim _{\mathrm{R} \rightarrow \infty} \mathrm{I}_{\Lambda_{\mathrm{a}, \mathrm{R}}}(\mathrm{g}, \mathrm{t}) \equiv \lim _{\mathrm{R} \rightarrow \infty} \int_{\mathrm{t}}^{\infty} \Phi_{\Lambda_{\mathrm{a}, \mathrm{R}}}\left(\mathrm{U}_{\tau} \mathrm{g}\right) \mathrm{d} \tau=\int_{\mathrm{t}}^{\infty} \Phi_{\Lambda_{\mathrm{a}}}\left(\mathrm{U}_{\tau} \mathrm{g}\right) \mathrm{d} \tau
$$

and

$$
\left|\int_{t}^{\infty} \Phi_{\Lambda_{a}}\left(U_{\tau} g\right) d \tau\right| \leq \int_{t}^{\infty} \eta(\tau) d \tau
$$

(ii) By using first (H1), then (12) and (10), we obtain

$$
\begin{aligned}
\left\|E\left(x \in C_{a}\right) U_{t} g\right\|^{2} & =\lim _{R \rightarrow \infty}\left\|E\left(x \in C_{a, R}\right) U_{t} g\right\|^{2}=-\lim _{R \rightarrow \infty} \int_{t}^{\infty} \frac{d}{d}\left\|E\left(x \in C_{a, R}\right) U_{\tau} g\right\|^{2} d \tau \\
& =\lim _{R \rightarrow \infty}\left\{I_{\Sigma_{a}}(g, t)+I_{\Sigma_{R}}(g, t)+I_{\Lambda_{a, R}}(g, t)\right\} .
\end{aligned}
$$

Since the first limit in (19) exists, by combining (19) with (17), we deduce that $\lim I_{\Sigma_{R}}(g, t)$ as $\mathrm{R} \rightarrow \infty$ exists if $\mathrm{t}>\mathrm{t}_{\mathrm{o}}$ and that

$$
\left\|\mathrm{E}\left(\mathrm{x} \in \mathrm{C}_{\mathrm{a}}\right) \mathrm{U}_{\mathrm{t} g} \mathrm{~g}\right\|^{2}=\mathrm{I}_{\Sigma_{\mathrm{a}}}(\mathrm{g}, \mathrm{t})+\lim _{\mathrm{R} \rightarrow \infty} \mathrm{I}_{\Sigma_{\mathrm{R}}}(\mathrm{g}, \mathrm{t})+\int_{\mathrm{t}}^{\infty} \Phi_{\Lambda_{\mathrm{a}}}\left(\mathrm{U}_{\tau} \mathrm{g}\right) \mathrm{d} \tau
$$

Now observe, by $(H 2)$, that $\lim _{R \rightarrow \infty} I_{\Sigma_{R}}(\mathrm{~g}, \mathrm{t})$ is independent of $\mathrm{t}$, say $\lim _{\mathrm{R} \rightarrow \infty} I_{\Sigma_{R}}(\mathrm{~g}, \mathrm{t})=\ell$. So the r.h.s. of (16) is equal to $\ell$. Finally, by taking into account $(\mathrm{H} 3)$ and the inequality (18), and by remembering that $\eta \in L^{1}\left(t_{0}, \infty\right)$, one finds from (20) that $\left\|E\left(x \in C_{a}\right) U_{t} g\right\|^{2}$ converges to $\ell$ as $\mathrm{t} \rightarrow+\infty$. It follows immediately that $0 \leq \ell \leq\|\mathrm{g}\|^{2}$. The second half of the proposition is a straightforward consequence.

Having proved (16) for all admissible cones, the result can easily be obtained for a more general class of cones by considering unions or differences of admissible cones. For example if $\mathrm{C}$ is a cone that can be subdivided by a finite number of smooth internal surfaces (in fact plane surfaces) into a union of admissible cones $\left\{C_{k}\right\}_{k=1}^{M}$, then (16) holds for $C$ because $\left\|\mathrm{E}\left(\mathrm{x} \in \mathrm{C}_{\mathrm{a}}\right) \mathrm{U}_{\mathrm{t}} \mathrm{g}\right\|^{2}=\Sigma_{\mathrm{k}=1}^{\mathrm{M}}\left\|\mathrm{E}\left(\mathbf{x} \in \mathrm{C}_{\mathrm{k}, \mathrm{a}}\right) \mathrm{U}_{\mathrm{t}} \mathrm{g}\right\|^{2}$ and, with the notation $\Sigma_{\mathrm{k}, \mathrm{R}}=\mathrm{C}_{\mathrm{k}} \cap \mathrm{S}_{\mathrm{R}}: \Phi_{\Sigma_{\mathrm{R}}}\left(\mathrm{U}_{\mathrm{t}} \mathrm{g}\right)=$ $\Sigma_{k=1}^{M} \Phi_{\Sigma_{k, R}}\left(U_{t} g\right)$. By a limiting procedure one could also treat certain cones that are subdivisible into a countable union of admissible cones.

Similarly one may pass from admissible cones to another class of non-convex cones: let $\mathrm{C}$ be an admissible cone and let $\left\{\mathrm{C}_{\mathrm{k}}\right\}_{\mathrm{k}=1}^{\mathrm{M}}$ be pairwise disjoint admissible cones contained in C. Let $\hat{C}=C \backslash\left(\cup_{k=1}^{M} C_{k}\right)$. Then, by arguing as above, (16) holds for $\hat{C}$. 
The above procedures allow us to treat all reasonably shaped cones, in particular circular cones $\left(\partial \Sigma_{\mathrm{a}}\right.$ is a circle), rectangular cones (the intersection of $\mathrm{C}$ with some plane not containing the origin is a rectangle) cones having holes (e.g. the cone corresponding to an annular array of counters, where $\Sigma_{R}$ may be the region between two circles on the sphere $S_{R}$ ).

\section{Estimates on the Flux for Smooth Potentials}

In this section we verify (16) in the case $\mathrm{U}_{\mathrm{t}}=\exp (-\mathrm{iHt})$ and $\mathrm{H}=\mathrm{H}_{\mathrm{O}}+\mathrm{V}(\mathbf{Q})$, where $\mathrm{V}$ is a smooth potential, for a dense subset of vectors $\mathrm{g}$ in the absolutely continuous subspace $\mathcal{H}_{\mathrm{ac}}(\mathrm{H})$ of the hamiltonian $\mathrm{H}$. We assume that $\mathrm{V}: \mathbb{R}^{3} \rightarrow \mathbb{R}$ is of class $\mathrm{C}^{\infty}$ and that for each multi-index $\alpha$ there is a constant $c_{\alpha}$ such that, for some $\kappa>0$ :

$$
\left|D^{\alpha} V(x)\right| \leq c_{\alpha}<x>^{-\kappa-|\alpha|} \equiv c_{\alpha}\left(1+x^{2}\right)^{-(\kappa+|\alpha|) / 2}
$$

Clearly in this case $D(H)=D\left(H_{0}\right)$. We shall use the following results concerning these hamiltonians:

(P1) The spectrum of $\mathrm{H}$ consists of an absolutely continuous part $\sigma_{a c}(H)=[0, \infty)$ and possibly also a set of eigenvalues in $\left(-c_{0}, 0\right]$, where $c_{o}$ denotes the constant $c_{\alpha}$ for $\alpha=0$. In particular $H$ has no singularly continuous spectrum and no positive eigenvalues.

(P2) If $\psi \in \mathrm{C}_{0}^{\infty}(\mathbb{R})$ and $\mu \geq 0$, then $\left\langle\mathrm{Q}>\mu^{\mu} \psi(\mathrm{H})<\mathrm{Q}>-\mu \in \mathbb{B}(\mathcal{H})\right.$.

(P3) If $z$ belongs to the resolvent set of $H, W \in L^{2}\left(\mathbb{R}^{3}\right)$ and $\mu \in \mathbb{R}$, then the closures of $<\mathrm{Q}>{ }^{-\mu} \mathrm{W}(\mathbf{Q})(\mathrm{H}-\mathrm{z})^{-1}<\mathrm{Q}>{ }^{\mu}$ and $<\mathrm{Q}>^{-}{ }^{-\mu} \mathrm{P}_{\mathrm{j}}(\mathrm{H}-\mathrm{z})^{-1}<\mathrm{Q}>^{\mu}$ belong to $\boldsymbol{B}(\mathcal{H})$.

(P4) Let $\psi \in C_{0}^{\infty}((0, \infty))$ and $0 \leq v^{\prime}<v$. Then there is a constant $c$ such that for all $t \in \mathbb{R}$ :

$$
\left\|<Q>^{-v} e^{-i H t} \psi(H)<Q>^{-v}\right\| \leq c(1+|t|)^{-v^{\prime}}
$$

For proofs of these results the reader may consult for example the following references: [12] for (P1), Lemmas 2 and 5 of [13] for (P2), Lemma 3 of [13] for (P3) and [14], [15] or [16] for (P4).

In what follows let $\omega$ be a fixed number satisfying $\omega>c_{0}+1$. Then $H+\omega>I$ and $\left\|(\mathrm{H}+\omega)^{-1}\right\|<1$. We shall use the following commutator identities:

$$
\begin{aligned}
{\left[\varphi(\mathbf{Q}),(\mathrm{H}+\omega)^{-1}\right] } & =(\mathrm{H}+\omega)^{-1}[\mathrm{H}, \varphi(\mathbf{Q})](\mathrm{H}+\omega)^{-1}=(\mathrm{H}+\omega)^{-1}\{(\Delta \varphi)(\mathbf{Q})-2 \mathrm{iP} \cdot(\nabla \varphi)(\mathbf{Q})\}(\mathrm{H}+\omega)^{-1} \\
& =(\mathrm{H}+\omega)^{-1}\{-(\Delta \varphi)(\mathbf{Q})-2 \mathrm{i}(\nabla \varphi)(\mathbf{Q}) \cdot \mathbf{P}\}(\mathrm{H}+\omega)^{-1}
\end{aligned}
$$


and (for suitable operators $A$ )

$$
\left[A, U_{t}\right]=-i U_{t} \int_{0}^{t} U_{-\tau}[A, H] U_{\tau} d \tau
$$

in cases where we use it, the formal relation (24) can be verified on an appropriate domain.

For $\rho \geq 0$ we let $\mathcal{D}_{\rho}(\mathrm{H})$ be the following subset of $\mathcal{H}$ :

$$
\mathcal{D}_{\rho}(\mathrm{H})=\left\{\psi(\mathrm{H})<\mathrm{Q}>^{-\rho_{\mathrm{f}}} \mid \psi \in \mathrm{C}_{0}^{\infty}((0, \infty)), \mathrm{f} \in \mathcal{H}\right)
$$

We shall often write $\mathscr{D}_{\rho}$ for $\mathscr{D}_{\rho}(\mathrm{H})$. One has $\mathscr{D}_{\rho} \subseteq \mathcal{H}_{\mathrm{ac}}(\mathrm{H}) \cap \mathrm{D}(\mathrm{H})$ and $\mathscr{D}_{\rho} \subseteq \mathrm{D}(<\mathrm{Q}>\rho)$ as a consequence of $(\mathrm{P} 1)$ and $(\mathrm{P} 2)$ respectively. Furthermore $U_{t} \mathscr{D}_{\rho} \subseteq \mathscr{D}_{\rho}$, and it is easy to check that $\mathscr{D}_{\rho}$ is dense in $\mathcal{H}_{\mathrm{ac}}(\mathrm{H})$. We also observe that $H \mathscr{D}_{\rho} \subseteq \mathfrak{D}_{\rho}$ and $\mathfrak{D}_{\mu} \subseteq \mathfrak{D}_{\rho}$ if $\mu \geq \rho$.

We shall use the following consequences of the propagation estimates (P4): Let $g \in \mathscr{D}_{\mathrm{v}}$, $0 \leq \nu^{\prime}<\nu$ and $\varphi: \mathbb{R}^{3} \rightarrow \mathbb{C}$ be such that $|\varphi(\mathbf{x})| \leq \mathrm{C}<\mathbf{x}>^{-v}$ for some constant $\mathrm{C}$. Observe that $\theta(\mathrm{H}) \mathrm{g}$ $=\mathrm{g}$ for some $\theta \in \mathrm{C}_{0}^{\infty}\left((0, \infty)\right.$ ) (choose $\theta$ such that $\theta u r=\psi$ if $g=\psi(\mathrm{H})<\mathrm{Q}>^{-v_{f}}$ ). So there is a constant $\mathrm{c}$ such that for all $t \in \mathbb{R}$ :

$$
\begin{gathered}
\left\|<Q>-v U_{t} g\right\| \leq c(1+|t|)^{-v}\left\|<Q>v^{\prime} g\right\| \\
\left\|\int_{0}^{t} \tau U_{-\tau} \varphi(Q) U_{\tau} g d \tau\right\| \leq c(1+|t|)^{2-v^{\prime}\left\|<Q>v^{v}\right\| .}
\end{gathered}
$$

Proposition 3 : Let $\mathrm{C}$ be an admissible cone. Assume that $V$ satisfies (21) with $\kappa>2 / 3$. Then (H1) - (H4) are satisfied for each $g \in \mathbb{D}_{\rho}(\mathrm{H})$ if $\rho>5 / 3$.

Since the proof of Proposition 3 is rather long, we split it up into a sequence of lemmas. Some of these require less stringent assumptions on $\kappa$ and $\rho$.

Lemma 2 : If $\kappa>0$ and $g \in \mathcal{H}_{\mathrm{ac}}(\mathrm{H})$, then $\left\|\mathrm{E}(|\mathbf{x}|<\mathrm{R}) \mathrm{U}_{\mathrm{t}} \mathrm{g}\right\| \rightarrow 0$ as $|\mathrm{t}| \rightarrow \infty$ for each $\mathrm{R}<\infty$. In particular (H1) is satisfied for each $\mathbf{g} \in \mathcal{H}_{\mathrm{ac}}(\mathrm{H})$.

Proof: If $g \in \mathcal{H}_{\mathrm{ac}}(\mathrm{H})$, then $w-\lim _{|t| \rightarrow \infty} U_{\mathrm{t}} \mathrm{g}=0$, and the result follows upon observing that $\mathrm{E}(|\mathbf{x}|<\mathrm{R})$ is $\mathrm{H}$-compact.

Lemma 3: If $\kappa>0$ and $g \in D(H)$, then the condition (H2) is satisfied.

Proof : By virtue of the Lebesgue dominated convergence theorem, it suffices to show that there is a constant $c<\infty$ such that $\left|\Phi_{\Sigma_{R}}\left(U_{t} g\right)\right| \leq c$ for all $R>1$ and all $t \in \mathbb{R}$ and that $\lim _{R \rightarrow \infty} \Phi_{\Sigma_{R}}\left(U_{t} g\right)=0$ for each fixed $t \in \mathbb{R}$. For this we observe that $\left\|F_{\Sigma_{R}}\right\| \leq 40$ for all $R>1$ (the 
numerical value of the preceding constant is of course irrelevant but can be obtained by using (8) and (5), the Schwarz inequality for the sum in (5) and the inequality $\left\|P(I+P)\left(I+P^{2}\right)^{-1}\right\|$ $<5 / 4$ ). So we have from (7) that, for $R>1$ :

$$
\begin{aligned}
\left|\Phi_{\Sigma_{R}}\left(U_{t} g\right)\right| & \leq 40\left\|(I+P) U_{t} g\right\|\left\|\left(I+H_{0}\right) U_{t} g\right\| \leq 50\left\|\left(I+H_{0}\right) U_{t} g\right\|^{2} \\
& \leq 50\left\|\left(I+H_{0}\right)(H+\omega)^{-1}\right\| 2\|(H+\omega) g\|^{2}
\end{aligned}
$$

which is finite and independent of $R$ and $t$ (we have used the inequality $\left.\left\|(I+P)\left(I+P^{2}\right)^{-1}\right\|<5 / 4\right)$.

Next we choose $\theta: \mathbb{R}^{3} \rightarrow[0,1]$ of class $C^{\infty}$ such that $\theta(x)=0$ if $|x|<1 / 4$ and $\theta(x)=1$ if $|x|>1 / 2$, and we set $\theta_{R}(x)=\theta(x / R)$, so that $\theta_{R} \in \Theta_{\Sigma_{R}}$. Then, by using (9), we obtain as above that

$$
\left|\Phi_{\Sigma_{R}}\left(U_{t} g\right)\right| \leq 50\left\|\left(I+H_{0}\right) \theta_{R}(Q) U_{t} g\right\|^{2}
$$

Now

$$
\left(\mathrm{I}+\mathrm{H}_{\mathrm{O}}\right) \theta_{\mathrm{R}}(\mathbf{Q})=\theta\left(\frac{\mathbf{Q}}{\mathrm{R}}\right)\left(\mathrm{I}+\mathrm{H}_{\mathrm{O}}\right)-\frac{1}{\mathrm{R}^{2}}(\Delta \theta)\left(\frac{\mathbf{Q}}{\mathrm{R}}\right)-\frac{2 \mathrm{i}}{\mathrm{R}}(\nabla \theta)\left(\frac{\mathbf{Q}}{\mathrm{R}}\right) \cdot \mathbf{P}
$$

When applied to a fixed vector in $\mathrm{D}\left(\mathrm{H}_{\mathrm{O}}\right)$, each term on the r.h.s. of (30) converges strongly to zero as $R \rightarrow \infty$. So $\Phi_{\Sigma_{R}}\left(U_{t} g\right) \rightarrow 0$ as $R \rightarrow \infty$ for each fixed $t \in \mathbb{R}$.

Lemma 4 : If $\kappa>0$ and $g \in \mathfrak{O}_{v}(\mathrm{H})$ with $v>1 / 2$, then $(\mathrm{H} 3)$ is satisfied.

Proof : Fix $v^{\prime} \in(1 / 2, v)$ and choose $\theta: \mathbb{R}^{3} \rightarrow[0,1]$ of class $C^{\infty}$ such that $\theta(x)=1$ if $|x|<a+1$ and $\theta(\mathbf{x})=0$ if $|\mathbf{x}|>a+2$. Then, as in the preceding proof:

$$
\begin{gathered}
\left|\Phi_{\Sigma_{\mathrm{a}}}\left(\mathrm{U}_{\mathrm{L}} \mathrm{g}\right)\right| \leq 4\left\|\Gamma_{\Sigma_{\mathrm{a}}}\right\|^{2}\left\|\left(\mathrm{I}+\mathrm{H}_{\mathrm{O}}\right) \theta(\mathbf{Q}) \mathrm{U}_{\mathrm{t}} \mathrm{g}\right\|^{2}= \\
=4\left\|\Gamma_{\Sigma_{\mathrm{a}}}\right\|^{2}\left\|\theta(\mathbf{Q}) \mathrm{U}_{\mathrm{t}} \mathrm{g}+\theta(\mathbf{Q})[\mathrm{H}-\mathrm{V}(\mathbf{Q})] \mathrm{U}_{\mathrm{t}} \mathrm{g}-(\Delta \theta)(\mathbf{Q}) \mathrm{U}_{\mathrm{t}} \mathrm{g}-2 \mathrm{i}(\nabla \theta)(\mathbf{Q}) \cdot \mathbf{P} \mathrm{U}_{\mathrm{t}} \mathrm{g}\right\|^{2} .
\end{gathered}
$$

Since the closures of $\theta(\mathbf{Q})<Q>v,(\Delta \theta)(\mathbf{Q})<Q>v^{v}$ and $\left(\partial_{j} \theta\right)(\mathbf{Q})<Q>v$ are bounded operators (with norm $\leq \hat{c}$, say), one gets from (26) the following bounds for the terms in the last norm:

$$
\begin{aligned}
& \left\|\theta(\mathbf{Q}) U_{\mathrm{t}} g-\theta(\mathbf{Q}) \mathrm{V}(\mathbf{Q}) \mathrm{U}_{\mathrm{t}} \mathrm{g}-(\Delta \theta)(\mathbf{Q}) \mathrm{U}_{\mathrm{t}} \mathrm{g}\right\| \leq \hat{\mathrm{c}} \mathrm{c}\left[2+\|\mathrm{V}\|_{\infty}\right](1+\mid \mathrm{tt})^{-v^{\prime}}\left\|<\mathrm{Q}>^{v} \mathrm{~g}\right\| \\
& \left\|\theta(Q) H U_{t} g\right\|=\left\|\theta(Q) U_{t} H g\right\| \leq \operatorname{const}(1+|t|)^{-v^{\prime}}, \\
& \left\|\left(\partial_{j} \theta\right)(Q) P_{j} U_{t} g\right\| \leq \hat{c}\left\|<Q>^{-v} P_{j}(H+\omega)^{-1}<Q>^{v}\right\|\left\|<Q>^{-v} U_{t}(H+\omega) g\right\| \leq \text { const }(1+|t|)^{-v},
\end{aligned}
$$

where we have used (26), (P3) and the fact that $\mathrm{Hg} \in \mathscr{D}_{\vee}$ if $g \in \mathscr{D}_{v}$. We conclude that $\left|\Phi_{\Sigma_{\mathrm{a}}}\left(\mathrm{U}_{\mathrm{t}} \mathrm{g}\right)\right| \leq \mathrm{const}(1+|\mathrm{t}|)^{-2 v^{\prime}}$, which implies (H3). 
It remains to prove the validity of $(\mathrm{H} 4)$ under the assumptions of Proposition 3. To do this, observe that, if $\theta$ is a conical 2-dimensional surface (in particular if $\theta=\theta_{k}$ ), then $\Sigma_{j=1}^{3} n_{j}(y) y_{j} \equiv n(y) \cdot y=0$ for $y \in \mathcal{O}$. In that case an alternative expression for $\Phi_{\Theta}(f)$ may be obtained as follows (cf. (7)):

$$
\begin{aligned}
\Phi_{\Theta}(f) & =2 \operatorname{Re} \Sigma_{j=1}^{3} \int_{\Theta} \overline{\left(\gamma_{\Theta} f\right)(y)} n_{j}(y)\left\{\gamma_{\Theta}\left(P_{j}-\lambda Q_{j}\right) f\right\}(y) d \sigma(y) \\
& =2 \operatorname{Re} \Sigma_{j=1}^{3}<(I+P) \theta(Q) f, \Gamma_{\Theta}^{*} n_{j} \Gamma_{\Theta}(I+P)\left(P_{j}-\lambda Q_{j}\right) f>
\end{aligned}
$$

if $\theta \in \Theta_{\Theta}$ and $\lambda$ is any number. Taking $f=U_{t} g$ and $\lambda=(2 t)^{-1}$, and recalling that $\Lambda_{a, R}=U_{k=1}^{N} \Theta_{k, R}$ modulo a finite number of straight lines, we have (with $\left\|\Gamma_{\Theta_{k, R}}\right\| \leq\left\|\Gamma_{\Lambda_{a}}\right\|$ )

$$
\left|\Phi_{\Lambda_{\mathrm{a}, \mathrm{R}}}\left(\mathrm{U}_{\mathrm{t}} \mathrm{g}\right)\right| \leq 2 \mathrm{~N}\left\|\Gamma_{\Lambda_{\mathrm{a}}}\right\|^{2}\left\|(\mathrm{I}+\mathrm{P}) \theta(\mathrm{Q}) \mathrm{U}_{\mathrm{t}} \mathrm{g}\right\| \Sigma_{\mathrm{j}=1}^{3}\left\|(\mathrm{I}+\mathrm{P})\left(\mathrm{P}_{\mathrm{j}}-\frac{\mathrm{Q}_{\mathrm{j}}}{2 \mathrm{t}}\right) \mathrm{U}_{\mathrm{t}} \mathrm{g}\right\|
$$

We shall find that $\left\|(I+P) \theta(Q) U_{t} g\right\| \leq$ const $(1+|t|)-\min \left(1 / 2, \kappa^{\prime} / 2\right)$ and $\left\|(I+P)\left(P_{j}-\frac{Q_{j}}{2 t}\right) U_{t} g\right\| \leq$ const $(1+|t|)^{-\min \left(1, \kappa^{\prime}\right)}$ for any $\kappa^{\prime}<\kappa$ and a suitable choice of $\theta$ in $\Theta_{\Lambda_{a}}$ (see Lemmas 8 and 6). This implies (H4) for $\kappa>2 / 3$.

Lemma 5 : Let $Y_{t}^{(j)}=2 \int_{0}^{t} \tau U_{-\tau}\left(\partial_{j} V\right)(Q) U_{\tau} d \tau$. Then for each $g \in \mathcal{D}_{1}$ one has, for $t>0$ :

$$
\left(P_{j}-\frac{Q_{j}}{2 t}\right) U_{t} g=-\frac{1}{2 t} U_{t}\left(Q_{j}+Y_{t}^{(j)}\right) g
$$

Proof : By Theorem 2.1 of [17] one has $U_{t g} g \in D\left(Q_{j}\right)$. From (24) it then follows that

$$
\left(P_{j}-\frac{Q_{j}}{2 t}\right) U_{t} g=U_{t} P_{j} g-U_{t} \int_{o}^{t} U_{-\tau}\left(\partial_{j} V\right)(Q) U_{\tau} g d \tau-\frac{1}{2 t} U_{t} Q_{j} g-\frac{1}{t} U_{t} \int_{o}^{t} U_{-\tau} P_{j} U_{\tau} g d \tau
$$

We deal with the last integral by commuting $P_{j}$ through $U_{\tau}$ (using (24) again) and then integrating by parts with respect to the $\tau$ variable. This gives

$$
\begin{aligned}
\int_{0}^{\mathrm{t}} U_{-\tau} P_{j} U_{\tau} g d \tau & =t P_{j} g-\int_{0}^{t} d \tau \int_{0}^{\tau} U_{-\sigma}\left(\partial_{j} V\right)(Q) U_{\sigma} g d \sigma \\
& =t_{j} g-\left.\tau \int_{0}^{\tau} U_{-\sigma}\left(\partial_{j} V\right)(Q) U_{\sigma} g d \sigma\right|_{\tau=0} ^{t}+\int_{0}^{t} \tau U_{-\tau}\left(\partial_{j} V\right)(Q) U_{\tau} g d \tau \\
& =\operatorname{tP} P_{j} g-t \int_{0}^{t} U_{-\sigma}\left(\partial_{j} V\right)(Q) U_{\sigma} g d \sigma+\frac{1}{2} Y_{t}^{(j)} g .
\end{aligned}
$$

Substituting this expression into (34), we arrive at (33). 
Lemma 6 : Suppose that $V$ satisfies (21) for some $\kappa>0$. For $g \in D(H)$, set $g_{\omega}=(H+\omega) g$. Then for each $\kappa^{\prime}<\kappa$ there is a constant $c=c\left(\kappa^{\prime}\right)$ such that for all $g \in \mathbb{D}_{\kappa+1}(H)$ :

$$
\left\|(I+P)\left(P_{j}-\frac{Q_{j}}{2 t}\right) U_{t} g\right\| \leq c(1+|t|)^{-m i n}\left(1, \kappa^{\prime}\right)\left[\left\|<Q>^{k+1} g\right\|+\left\|<Q>^{k+1}(H+\omega) g\right\|\right]
$$

Proof : (i) Let $Y_{t}^{(j)}$ be as in Lemma 5. The norm of $Y_{t}^{(j)} g$ may be estimated using (27) with $\varphi$ $=\partial_{\mathrm{j}} \mathrm{V}$ and $\mathrm{v}=\mathrm{K}+1$ :

$$
\left\|Y_{t}^{(j)} g\right\| \leq c_{j}(1+|t|)^{1-k^{\prime}}\left\|<Q>^{k+1} g\right\|
$$

Here $c_{j}$ is a constant depending also on $\kappa^{\prime}$ (note that $\left\|Y_{t}^{(j)}\right\|$ is uniformly bounded in $t$ if $V$ is a short range potential, i.e. in the case $\kappa>1$ ).

(ii) From (33) and (36) we now have:

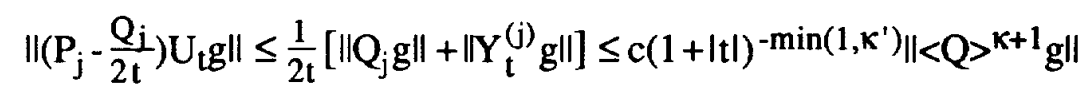

and

$$
\left\|P_{k}\left(P_{j}-\frac{Q_{j}}{2 t}\right) U_{t} g\right\| \leq \frac{1}{2 t}\left[\left\|P_{k} U_{t} Q_{j}(H+\omega)^{-1} g_{\omega}\right\|+\left\|P_{k} U_{t} Y_{t}^{(j)}(H+\omega)^{-1} g_{\omega}\right\|\right]
$$

The norms on the r.h.s. of (38) are handled by commuting $(H+\omega)^{-1}$ through $Q_{j}$ and $Y_{t}^{(j)}$ respectively (using (23)), to give in the first instance

$$
\left\|P_{k} U_{t} Q_{j}(H+\omega)^{-1} g_{\omega}\right\| \leq\left\|P_{k}(H+\omega)^{-1}\right\|\left\|Q_{j} g_{\omega}\right\|+\left\|P_{k}(H+\omega)^{-1}\right\|\left\|\left[Q_{j}, H\right] g\right\|
$$

Since $\left[Q_{j}, H\right]=2 i P_{j}$ and $P_{k}(H+\omega)^{-1} \in ß(\mathcal{H})$, the r.h.s. of this inequality is finite. For the following norm we have

$$
\begin{aligned}
& \left\|P_{k} U_{t} Y_{t}^{(j)}(H+\omega)^{-1} g_{\omega}\right\| \leq\left\|P_{k}(H+\omega)^{-1}\right\|\left\|Y_{t}^{(j)} g_{\omega}\right\|+ \\
& \quad+2\left\|P_{k}(H+\omega)^{-1 / 2}\right\|\left\|(H+\omega)^{-1 / 2} \int_{0}^{t} \tau d \tau U_{-\tau}\left[\left(\partial_{j} \Delta V\right)(Q)-2 i \Sigma_{\ell=1}^{3} P_{\ell}\left(\partial_{j} \partial_{\ell} V\right)(Q)\right] U_{\tau} g\right\|
\end{aligned}
$$

By (36) the first summand on the r.h.s. is $\leq$ const $(1+|t|)^{1-\kappa^{\prime}}\left\|<\mathrm{Q}>\boldsymbol{k + 1} \mathrm{g}_{\omega}\right\|$. Since $\mathrm{P}_{\mathbf{k}}(\mathrm{H}+\omega)^{-1 / 2}$ $=\mathrm{P}_{\mathrm{k}}\left(\mathrm{H}_{\mathrm{O}}+\omega\right)^{-1 / 2} \cdot\left(\mathrm{H}_{\mathrm{O}}+\omega\right)^{1 / 2}(\mathrm{H}+\omega)^{-1 / 2}$ is bounded, the second summand on the r.h.s. is bounded by a constant multiple of

$$
\int_{0}^{t} \tau d \tau\left\|\left(\partial_{j} \Delta V\right)(Q) U_{\tau} g\right\|+2 \Sigma_{\ell=1}^{3}\left\|P_{\ell}(H+\omega)^{-1 / 2}\right\| \cdot \int_{0}^{t} \tau d \tau\left\|\left(\partial_{j} \partial_{\ell} V\right)(Q) U_{\tau} g\right\| .
$$

With $\left|\left(\partial_{j} \Delta V\right)(x)\right|+\left|\left(\partial_{j} \partial_{\ell} V\right)(x)\right| \leq c<x>^{-k-2} \leq c<x>^{-k-1}$, we see from (26) that the preceding sum is $\leq$ const $(1+|\mathrm{t}|)^{1-\kappa^{\prime} \|<Q>^{K+1}} \mathrm{~g} \|$. 
Putting together the above estimates, one arrives at (35). (Observe that $\left\|\mathrm{P}_{\mathrm{j}} \mathrm{g}\right\| \leq$ $c\|(H+\omega) g\|$ and recall that $\left.\|\mathrm{Pf}\|^{2}=\Sigma_{\mathrm{j}=1}^{3}\|\mathrm{P} \mathrm{j}\|^{2}\right)$.

Lemma 7 : Let $C$ be an admissible cone, $\xi$ a unit vector in the interior of $C$ and $\sigma>0$. Let $\mathrm{C}(\sigma)=\left\{\mathbf{x} \in \mathbb{R}^{3} \mid \mathbf{x}=\mathbf{y}+\mu \xi, \mathbf{y} \in \partial \mathrm{C},-\sigma<\mu<\sigma\right\}$ be the domain in $\mathbb{R}^{3}$ obtained by translating $\partial \mathrm{C}$ along $\xi$ by distances less than $\sigma$. Let $\beta>1 / 2$. Then there is a finite constant $c$ such that for each bounded function $\varphi: \mathbb{R}^{3} \rightarrow \mathbb{C}$ with support in $C(\sigma)$ and for all $t \in \mathbb{R}$ (with $U_{t}^{o}=\exp \left(-\mathrm{iH}_{0} t\right)$ ):

$$
\left\|\varphi(\mathbf{Q}) \mathrm{U}_{\mathrm{t}}^{\mathrm{O}}(\mathbf{I}+|\xi \cdot \mathbf{Q}|)^{-\beta}\right\| \leq \mathrm{c}\|\varphi\|_{\infty}(1+|\mathrm{t}|)^{-1 / 2}
$$

Proof : We choose a cartesian coordinate system such that the 3 -axis is along $\xi$, so that $\xi \cdot \mathbf{Q}=$ $\mathrm{Q}_{3}$. We shall use the Dollard decomposition of $\exp \left(-\mathrm{iP}_{3}^{2} \mathrm{t}\right)$, given for $\mathrm{t}>0$ by $\exp \left(-\mathrm{iP}_{3}^{2} \mathrm{t}\right)=$ $\mathrm{Z}_{\mathrm{t}} \mathrm{G}_{\mathrm{t}} \mathrm{Z}_{\mathrm{t}}$ with

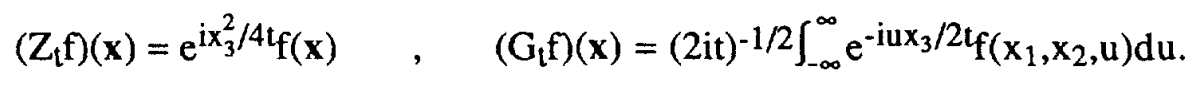

Since $P_{1}$ and $P_{2}$ commute with $Q_{3}$, we have

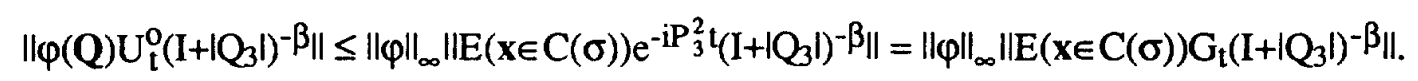

For fixed $x_{1}, x_{2}$, let $S\left(x_{1}, x_{2}\right)=\left\{x_{3} \in \mathbb{R} \mid x \equiv\left(x_{1}, x_{2}, x_{3}\right) \in C(\sigma)\right\}$ be the set of points in $C(\sigma)$ on the line parallel to the 3 -axis passing through the point $\left(x_{1}, x_{2}, 0\right)$. Since $C$ is convex, $S\left(x_{1}, x_{2}\right)$ is an interval of length $2 \sigma$. So, by using the Schwarz inequality in the integral with respect to $u$ below, we have for $t>0$ :

$$
\begin{aligned}
& \|\left. E(x \in C(\sigma)) G_{t}\left(I+\left|Q_{3}\right|\right)^{-\beta} f^{2}\right|^{2}=\frac{1}{2 t} \int_{-\infty}^{\infty} d_{x_{1}} \int_{-\infty}^{\infty} d x_{2} \int_{S\left(x_{1}, x_{2}\right)} d x_{3}\left|\int_{-\infty}^{\infty} e^{-i u x_{3} / 2 t}(1+|u|)^{-\beta} f\left(x_{1}, x_{2}, u\right) d u\right|^{2} \\
& \leq\left.\frac{1}{2 t} \int_{-\infty}^{\infty} d x_{1} \int_{-\infty}^{\infty} d_{x_{2}} \int_{S\left(x_{1}, x_{2}\right)} d x_{3} \int_{-\infty}^{\infty} d u l f\left(x_{1}, x_{2}, u\right)\right|^{2}\left\|(1+|x|)^{-\beta}\right\|_{L}^{2}(\mathbb{R}) \\
& =\frac{\sigma}{t}\|f\|^{2}\left\|(1+|x|)^{-\beta}\right\|_{L}^{2}(\mathbb{R}) .
\end{aligned}
$$

This implies (39) with $c=\left\{1+\sigma\left\|(1+|x|)^{-\beta}\right\|_{L}^{2}(\mathbb{R})\right\}^{1 / 2}$.

If $\mathrm{C}$ is an admissible cone, then $\mathrm{C}(\sigma)$ forms a neighbourhood of $\Lambda_{\mathrm{a}}$. In particular there are functions in $\Theta_{\Lambda_{\mathrm{a}}}$ having support in $\mathrm{C}(\sigma)$. Then we have 
Lemma 8 : Let $C$ be an admissible cone and $\theta$ a function in $\Theta_{\Lambda_{a}}$ with support in $C(\sigma)$ for some $\sigma>0$. Suppose that $V$ satisfies (21) for some $k>0$, and let $0 \leq \kappa^{\prime}<\kappa$. Then there is a finite constant c such that for each $g \in \mathscr{D}_{\kappa+1}(\mathrm{H})$ :

$$
\left\|(\mathrm{I}+\mathrm{P}) \theta(\mathbf{Q}) \mathrm{U}_{\mathrm{t}} \mathrm{g}\right\| \leq \mathrm{c}(1+|\mathrm{t}|)^{-\min \left(1 / 2, \mathrm{~K}^{\prime} / 2\right)}\left[\left\|<\mathrm{Q}>^{\mathrm{K}+1} \mathrm{~g}\right\|+\left\|<\mathrm{Q}>^{\mathrm{K}+1}(\mathrm{H}+\omega) \mathrm{g}\right\|\right] .
$$

Proof : The proof will introduce two parameters $\beta$ and $\kappa_{0}$, with $1 / 2<\beta \leq 1$ and $\kappa_{0}<\kappa$, the appropriate values of $\beta$ and $\kappa_{0}$ to be determined later.

(i) We begin with an auxiliary estimate. Using the relation $\left[Q_{j}, U_{-t}^{o}\right]=-2 t U_{-t}^{o} P_{j}$ and the bound (37), we have, for some constant c:

$$
\left\|Q_{j} U_{-t}^{o} U_{t} g\right\|=\left\|2 t U_{-t}^{o}\left(P_{j}-\frac{Q_{j}}{2 t}\right) U_{t} g\right\| \leq c(1+|t|)^{\max \left(0,1-k_{o}\right)}\left\|<Q>^{K+1} g\right\| .
$$

Hence, for any unit vector $\xi$, we have

$$
\left\|\xi \cdot Q \mid U_{-t}^{o} U_{t} g\right\|\left\|\leq \Sigma_{j=1}^{3}\right\| Q_{j} U_{-t}^{O} U_{t} g \| \leq 3 c(1+|t|)^{\max \left(0,1-\kappa_{o}\right)_{\|<Q}>^{\kappa+1} g \|,}
$$

and by interpolation:

$$
\left\|\left.\xi \cdot Q\right|^{\beta} U_{-t}^{o} U_{t} g\right\| \leq \operatorname{const}(1+|t|){ }^{\max \left(0, \beta-\beta \kappa_{o}\right)}\left\|<Q>^{K+1} g\right\|
$$

(ii) Now let $\xi$ be as in Lemma 7. From the inequalities $\left(a_{1}+a_{2}\right)^{\beta} \leq a_{1}^{\beta}+a_{2}^{\beta}$ for $a_{1}, a_{2} \geq 0,0<\beta \leq 1$ and from (39), (42) we find that

$$
\begin{aligned}
& \left\|\theta(\mathbf{Q}) \mathrm{U}_{\mathrm{t}} \mathrm{g}\right\| \leq\left\|\theta(\mathbf{Q}) \mathrm{U}_{\mathrm{t}}^{\mathrm{O}}(\mathrm{I}+|\xi \cdot \mathrm{Q}|)^{-\beta}\right\|\left\|(\mathrm{I}+|\xi \cdot \mathrm{Q}|)^{\beta} \mathrm{U}_{-\mathrm{t}}^{\mathrm{o}} \mathrm{U}_{\mathrm{t}} \mathrm{g}\right\| \\
& \leq \mathrm{c}\|\theta\|_{\infty}(1+|\mathrm{t}|)^{-1 / 2}\left[\|\mathrm{~g}\|+\left\||\xi \cdot \mathrm{Q}|^{\beta} \mathrm{U}_{-\mathrm{t}}^{\mathrm{o}} \mathrm{U}_{\mathrm{t}} \mathrm{g}\right\|\right] \leq \tilde{\mathrm{c}}(1+|\mathrm{t}|)^{-\min \left(1 / 2,1 / 2-\beta+\beta \kappa_{\mathrm{o}}\right)_{\|<Q}>^{\mathrm{K}+1} \mathrm{~g} \| .}
\end{aligned}
$$

Now choose $\beta$ and $\kappa_{0}$ such that the preceding estimate agrees with that shown in (40). If $\kappa^{\prime} \geq 1$, take $\beta=1$ and $\kappa_{0}=\kappa^{\prime}$; then $\min \left(1 / 2,1 / 2-\beta+\beta \kappa_{0}\right)=1 / 2=\min \left(1 / 2, \kappa^{\prime} / 2\right)$. If $\kappa^{\prime}<1$, take $\kappa_{0}=1 / 2\left[\kappa^{\prime}+\min (1, \kappa)\right]$ and $\beta=1 / 2\left(1-\kappa^{\prime}\right)\left(1-\kappa_{0}\right)^{-1}$. Then $\beta \in(1 / 2,1], \kappa_{0} \in\left(\kappa^{\prime}, \min (1, \kappa)\right)$ and $1 / 2-\beta+\beta \kappa_{0}=\kappa^{\prime} / 2$.

(iii) It remains to carry out similar estimates for $\left\|P \theta(Q) U_{t} g\right\| \leq \Sigma_{j=1}^{3}\left\|P_{j} \theta(Q) U_{t} g\right\|$. Writing $g=$ $(\mathrm{H}+\omega)^{-1} \mathrm{~g}_{\omega}$ and commuting $\theta(\mathrm{Q})$ through $(\mathrm{H}+\omega)^{-1}$, as in the proof of Lemma 6 , gives

$$
\begin{aligned}
\left\|P_{j} \theta(\mathbf{Q}) U_{\mathrm{t}} g\right\| \leq & \left\|\mathrm{P}_{\mathrm{j}}(\mathrm{H}+\omega)^{-1} \theta(\mathbf{Q}) \mathrm{U}_{\mathrm{t}} \mathrm{g}_{\omega}\right\|+\left\|\mathrm{P}_{\mathrm{j}}(\mathrm{H}+\omega)^{-1}\left[(\Delta \theta)(\mathbf{Q})-2 \mathrm{i} \Sigma_{\ell=1}^{3} \mathrm{P}_{\ell}\left(\partial_{\ell} \theta\right)(\mathbf{Q})\right] \mathrm{U}_{\mathrm{t}} \mathrm{g}\right\| \\
& \leq\left\|\mathrm{P}_{\mathrm{j}}(\mathrm{H}+\omega)^{-1}\right\|\left[\left\|\theta(\mathbf{Q}) \mathrm{U}_{\mathrm{t}} \mathrm{g}_{\omega}\right\|+\left\|(\Delta \theta)(\mathbf{Q}) \mathrm{U}_{\mathrm{t}} \mathrm{g}\right\|\right]+ \\
& +2\left\|\mathrm{P}_{\mathrm{j}}(\mathrm{H}+\omega)^{-1 / 2}\right\| \Sigma_{\ell=1}^{3}\left\|\mathrm{P}_{\ell}(\mathrm{H}+\omega)^{-1 / 2}\right\|\left\|\left(\partial_{\ell} \theta\right)(\mathbf{Q}) \mathrm{U}_{\mathrm{t}} \mathrm{g}\right\| .
\end{aligned}
$$


Since supp $\Delta \theta \subset \mathrm{C}(\sigma)$ and supp $\partial_{\ell} \theta \subset \mathrm{C}(\sigma)$, the result of (ii) (first for the function $\theta$, then with $\theta$ replaced by $\Delta \theta$ and by $\partial_{\ell} \theta$ respectively) allows us to majorize the above expression by

$$
\text { const }(1+|t|)^{-\min \left(1 / 2, \kappa^{\prime} / 2\right)}\left[\left\|<Q>^{\kappa+1} g\right\|+\left\|<Q>^{\kappa+1} g_{\omega}\right\|\right] \text {. }
$$

Remarks : (a) Lemmas 6 and 8 lead to estimates of $\Phi_{\Lambda_{\mathrm{a}}}\left(\mathrm{U}_{\mathrm{t}} \mathrm{g}\right)$ in terms of $\left\|<Q>^{\mathrm{K}+1} \mathrm{~g}\right\|$ and $\left\|<Q>{ }^{k+1}(H+\omega) g\right\|$, whereas in Proposition 3 we required only that $g \in \mathscr{D}_{\rho}$ for some $\rho>5 / 3$ (so $\rho$ could be less than $\kappa+1$ if $\kappa$ is large). To see that the condition $g \in \mathscr{D}_{\rho}$ for some $\rho>5 / 3$ is sufficient, it is enough to observe that, if $V$ satisfies (21) for some $\kappa$, then it also satisfies these inequalities with $\kappa$ replaced by $\min (\kappa, \rho-1)$, in which case Lemmas 6 and 8 may be applied with $\kappa$ replaced by $\min (\kappa, \rho-1)$.

(b) If $\mathrm{V}$ is a short range potential, so that $\mathrm{k}>1$, we may take $\kappa^{\prime}>1$ in Lemmas 6 and 8 and deduce that $\left|\Phi_{\Lambda_{\mathrm{a}, \mathrm{R}}}\left(\mathrm{U}_{\mathrm{t}} \mathrm{g}\right)\right| \leq \mathrm{c}(1+|\mathrm{t}|)^{-3 / 2}$.

(c) Proposition 2 was formulated in terms of a limit $\mathrm{t} \rightarrow+\infty$. It is clear, under the assumptions on $\mathrm{V}$ and $\mathrm{g}$ stated in Proposition 3, that a similar result holds for the limit of negative times, namely

$$
\lim _{t \rightarrow-\infty}\left\|E\left(x \in C_{a}\right) U_{L} g\right\|^{2}=-\lim _{R \rightarrow \infty} \int_{-\infty}^{T} \Phi_{\Sigma_{R}}\left(U_{t} g\right) d t
$$

\section{The Flux for Singular Potentials}

In this section we shall extend the results of Proposition 3 to a class of potentials having local singularities. We consider potentials $\mathrm{V}$ that may be expressed in the form $V=V_{1}+V_{2}+V_{3}$, where $V_{1}$ is smooth, $V_{2}$ contains square-integrable local singularities and $V_{3}$ may have arbitrary singularities on a bounded closed set $\zeta$ of measure zero in $\mathbb{R}^{3}$ (a specially important case is that in which $\xi=\{0\}: V_{3}$ may then have an arbitrary singularity at the origin). If $\mathrm{V}_{3} \neq 0$, the hamiltonian need not be uniquely defined as a self-adjoint operator.

Our assumptions on $\mathrm{V}=\mathrm{V}_{1}+\mathrm{V}_{2}+\mathrm{V}_{3}$ are as follows: $\mathrm{V}_{\mathrm{k}}(\mathrm{k}=1,2,3)$ are real-valued functions on $\mathbb{R}^{3}$ such that

(V1) $V_{1}$ is of class $C^{\infty}$ and satisfies (21) for some $k>0$ (later we shall assume $k>2 / 3$ as before),

(V2) $\left(1+x^{2}\right)^{\sigma / 2} V_{2} \in L^{2}\left(\mathbb{R}^{3}\right)$ for some $\sigma>2$,

(V3) there is a number $r_{0}>0$, and a closed set $\zeta$ of measure zero, with $\zeta \subset B_{r_{0}} \equiv$ $\left\{\mathbf{x} \in \mathbb{R}^{3}|| \mathbf{x} \mid \leq r_{o}\right\}$, such that $\mathrm{V}_{3}(\mathbf{x})=0$ if $|\mathbf{x}|>\mathrm{r}_{\mathrm{o}}$ and $\mathrm{V}_{3} \in \mathrm{L}_{l \circ \mathrm{c}}^{2}\left(\mathrm{~B}_{\mathrm{r}_{\mathrm{o}}} \mid \mathrm{G}\right)$. 
We set $\mathrm{H}_{1}=\mathrm{H}_{0}+\mathrm{V}_{1}(\mathbf{Q})$ and let $\mathrm{H}$ be an arbitrary self-adjoint extension of the symmetric operator $\hat{H}=H_{0}+V_{1}(\mathbf{Q})+V_{2}(\mathbf{Q})+V_{3}(\mathbf{Q})$ defined on $s_{\zeta}=\left\{f \in B\left(\mathbb{R}^{3}\right) \mid f=0\right.$ in some neighbourhood of $\xi_{\}}$. If $V_{3}=0$, we take $H$ to be the unique self-adjoint operator $H_{0}+V_{1}(Q)+V_{2}(Q)$ defined on $D\left(H_{0}\right)$, i.e. $D(H)=D\left(H_{0}\right)$. If $V_{3} \neq 0$, the domain of $H$ (for any self-adjoint extension of $\hat{\mathrm{H}}$ ) coincides with that of $\mathrm{H}_{0}$ away from the set $\xi$, in the sense that if $\varphi: \mathbb{R}^{3} \rightarrow \mathbb{C}$ is of class $C^{\infty}$, such that $\varphi, \partial_{j} \varphi$ and $\Delta \varphi$ are bounded and $\varphi=0$ in some neighbourhood of $\zeta$, then

$$
\mathrm{f} \in \mathrm{D}(\mathrm{H}) \Rightarrow \varphi(\mathbf{Q}) \mathrm{f} \in \mathrm{D}\left(\mathrm{H}_{\mathrm{O}}\right) \cap \mathrm{D}(\mathrm{V}(\mathbf{Q})) \cap \mathrm{D}(\mathrm{H}) \text { and } \mathrm{H} \varphi(\mathbf{Q}) \mathrm{f}=\mathrm{H}_{\mathrm{o}} \varphi(\mathbf{Q}) \mathrm{f}+\mathrm{V}(\mathbf{Q}) \varphi(\mathbf{Q}) \mathrm{f}
$$

In particular:

$$
H_{0} \varphi(\mathbf{Q})(\mathbf{H}+\mathbf{i})^{-1} \in \mathbb{B}(\mathcal{H}),
$$

see for example Lemma 2 of [18] or Proposition 2.34 and Corollary 2.35 of [1] (the proofs given in [1] can easily be adapted to the present situation).

In order to deal with the singularities of $V_{3}$, we introduce a cut-off function $\eta \in C^{\infty}\left(\mathbb{R}^{3}\right)$ as follows: fix $a>r_{0}$ and let $\eta(x)=1$ if $|x|>a-\left(a-r_{0}\right) / 3, \eta(x)=0$ if $|x|<r_{0}+\left(a-r_{0}\right) / 3$ and $0 \leq \eta(x)$ $\leq 1$ otherwise. It is clear that $\eta \in \Theta_{\partial C_{a}}$ and that $H_{0} \eta(\mathbf{Q})(H+i)^{-1} \in \mathbb{B}(\mathcal{H})$. Also $V_{3}(\mathbf{Q}) \eta(\mathbf{Q})=0$.

Let $\mathrm{U}_{\mathrm{t}}=\exp (-\mathrm{iHt})$ and $\mathrm{U}_{\mathrm{t}}^{1}=\exp \left(-\mathrm{iH}_{1} \mathrm{t}\right)$. We note that all results obtained in Section 5 are valid for $U_{t}^{1}$. We shall use the relative wave operators $W_{ \pm}=s-\lim _{t \rightarrow \pm \infty} U_{-t} U_{t}^{1} E_{a c}\left(H_{1}\right)$, where $E_{a c}\left(H_{1}\right)$ is the orthogonal projection in $\mathcal{H}$ with range $\mathcal{H}_{a c}\left(H_{1}\right)$. The limits defining $W_{ \pm}$ exist if $V$ satisfies (V1)-(V3), and $W_{ \pm}$map $\mathcal{H}$ into $\mathcal{H}_{a c}(H)$. If $V_{3} \neq 0, W_{ \pm}$need not be complete, i.e. their ranges need not be equal to $\mathcal{H}_{\mathrm{ac}}(\mathrm{H})$. This point will be discussed at the end of this section.

Since $I-\eta(\mathbf{Q})$ is $\mathrm{H}_{1}$-compact, i.e. $[I-\eta(\mathbf{Q})]\left(\mathrm{H}_{1}+\mathbf{i}\right)^{-1}$ is a compact operator in $\mathcal{H}$, one has $s-\lim _{t \rightarrow \pm \infty}[I-\eta(Q)] U_{t}^{1} E_{a c}\left(H_{1}\right)=0$, so that

$$
W_{ \pm}=s-\lim _{t \rightarrow \pm \infty} U_{-t} U_{t}^{1} E_{a c}\left(H_{1}\right)=s-\lim _{t \rightarrow \pm \infty} U_{-t} \eta(Q) U_{t}^{1} E_{a c}\left(H_{1}\right)
$$

We shall need the following estimate:

Lemma 9 : Suppose that $V$ satisfies (V1)-(V3). Let $f \in \mathscr{O}_{v}\left(H_{1}\right)$ for some $v>1$, and let $1<v^{\prime}<\min (v, \sigma)$. Then there is a constant $\mathrm{c}<\infty$ such that for all $\mathrm{t}>0$ :

$$
\left\|U_{t} W_{+} f-\eta(Q) U_{t}^{1} f\right\|+\left\|H_{0} \eta(Q)\left[U_{t} W_{+} f-\eta(Q) U_{t}^{1} f\right]\right\| \leq c(1+|t|)^{1-v^{\prime}}
$$

Remark : The inequality $\| \frac{d}{d t} U_{-t} \eta(Q) U_{t}^{1} f l l \leq c(1+|t|)^{-v^{\prime}}$ obtained in the proof below implies in particular the existence of $W_{ \pm}$. 
Proof : We set $f_{i}=\left(H_{1}+i\right) f$.

(i) Taking note of (44) with $\varphi=\eta$, we obtain

$$
\begin{aligned}
\left\|U_{t} W_{+} f-\eta(Q) U_{t}^{1} f\right\| & =\left\|W_{+} f-U_{-t} \eta(Q) U_{t}^{1} f\right\|=\left\|\int_{t}^{\infty} \frac{d}{d \tau} U_{-\tau} \eta(Q) U_{\tau}^{1} f d \tau\right\| \\
& \leq \int_{t}^{\infty}\left\|\left[H \eta(Q)-\eta(Q) H_{1}\right] U_{\tau}^{1} f\right\| d \tau
\end{aligned}
$$

Now

$$
\left\|\left[H \eta(\mathbf{Q})-\eta(\mathbf{Q}) H_{1}\right] U_{\tau}^{1} f\right\|=\left\|\left[-(\Delta \eta)(\mathbf{Q})-2 i(\nabla \eta)(\mathbf{Q}) \cdot \mathbf{P}+\mathrm{V}_{2}(\mathbf{Q}) \eta(\mathbf{Q})\right] \mathbf{U}_{\tau}^{1} f\right\| .
$$

As in the proof of Lemma 4, we have for any $v^{\prime}<v$ :

$$
\left\|(\Delta \eta)(Q) U_{\tau}^{1} f\right\|+\left\|\left(\partial_{j} \eta\right)(Q) P_{j} U_{\tau}^{1} f\right\| \leq \operatorname{const}(1+|\tau|)^{-v^{\prime}}
$$

Furthermore, if $V_{2} \in L^{2}\left(\mathbb{R}^{3}\right)$ is defined as $V_{2}(x)=\left\langle x>\sigma V_{2}(x)\right.$ and $\left(H_{1}+i\right) f=f_{i}$, then

$$
\left\|V_{2}(Q) \eta(Q) U_{\tau}^{1} f\right\| \leq\left\|<Q>-\sigma V_{2}(Q)\left(H_{1}+\mathrm{i}\right)^{-1}<Q>\sigma_{\|\|<Q}>^{-\sigma} U_{\tau}^{1} f_{i}\right\|
$$

The finiteness of the first norm on the r.h.s. follows from property (P3) in Section 5, and by (26) the second norm does not exceed const $(1+|\tau|)^{-v^{\prime}}$ if $v^{\prime}<\min (v, \sigma)$. So we have for $t>0$ :

$$
\left\|\left[\mathrm{H} \eta(\mathbf{Q})-\eta(\mathbf{Q}) \mathrm{H}_{1}\right] \mathrm{U}_{\tau}^{1} \mathrm{f}\right\| \leq \mathrm{const}(1+|\tau|)^{-v^{\prime}}
$$

Substituting into (48) gives the bound for the first term on the left-hand side of (47).

(ii) From the intertwining property $\psi(\mathrm{H}) \mathrm{W}_{+}=\mathrm{W}_{+} \psi\left(\mathrm{H}_{1}\right)$, we have

$$
\begin{aligned}
& H_{0} \eta(Q)\left[U_{t} W_{+} f-\eta(Q) U_{t}^{1} f\right]=H_{o} \eta(Q)\left[(H+i)^{-1} U_{t} W_{+} f_{i}-\eta(Q)\left(H_{1}+i\right)^{-1} U_{t}^{1} f_{i}\right] \\
= & H_{o} \eta(Q)(H+i)^{-1}\left[U_{t} W_{+} f_{i}-\eta(Q) U_{t}^{1} f_{i}\right]+H_{o} \eta(Q)\left[(H+i)^{-1} \eta(Q)-\eta(Q)\left(H_{1}+i\right)^{-1}\right] U_{t}^{1} f_{i} .
\end{aligned}
$$

As $H_{0} \eta(Q)(H+i)^{-1} \in \mathcal{B}(\mathcal{H})$, the norm of the first summand on the r.h.s. is $\leq$ const $(1+|t|)^{1-v^{\prime}}$ by the result of (i). The second summand may be rewritten in the form

$$
\mathrm{H}_{\mathrm{O}} \eta(\mathbf{Q})(\mathrm{H}+\mathrm{i})^{-1}\left[\eta(\mathbf{Q}) \mathrm{H}_{1}-\mathrm{H} \eta(\mathbf{Q})\right]\left(\mathrm{H}_{1}+\mathrm{i}\right)^{-1} \mathrm{U}_{\mathrm{t}}^{1} \mathrm{f}_{\mathrm{i}}=\mathrm{H}_{\mathrm{O}} \eta(\mathbf{Q})(\mathrm{H}+\mathrm{i})^{-1}\left[\eta(\mathbf{Q}) \mathrm{H}_{1}-\mathrm{H} \eta(\mathbf{Q})\right] \mathrm{U}_{\mathrm{t}}^{1} \mathrm{f}
$$

so that by (49) the norm of this expression is bounded by const $(1+|t|)^{-v^{\prime}}$. These bounds may be used to estimate the second term on the l.h.s. of (47).

Theorem : Let $\mathrm{C}$ be an admissible cone. Suppose that the potential V satisfies (V1)-(V3) with $\kappa>2 / 3$ in $(\mathrm{V} 1)$. Then $(\mathrm{H} 1)-(\mathrm{H} 4)$ are satisfied for each vector $g$ of the form $g=W_{+} f$ provided $f \in \mathscr{D}_{\rho}\left(\mathrm{H}_{1}\right)$ for some $\rho>2$. For these vectors $g$ the limits in (16) exist and are finite and equal. 
Proof : We first prove (H1)-(H4) by using the following expression for the flux:

$$
\Phi_{\Theta}\left(U_{t} g\right)=\operatorname{Re}<(I+P) \eta(Q) U_{t} g, F_{Q}\left(I+H_{0}\right) \eta(Q) U_{t} g>
$$

where $\mathcal{Q}=\Sigma_{\mathrm{a}}, \Sigma_{\mathrm{R}}, \Lambda_{\mathrm{a}, \mathrm{R}}$ or $\partial \mathrm{C}_{\mathrm{a}, \mathrm{R}}$. We shall justify (50) in part (iv) of the proof.

(i) We have $f \in \mathcal{H}_{a c}\left(H_{1}\right)$, so that $g=W_{+} f \in \mathcal{H}_{a c}(H)$. To obtain (H1), it is enough to observe that $\mathrm{E}\left(\mathbf{x} \in \mathrm{C}_{\mathrm{a}, \mathrm{R}}\right)(\mathrm{H}+\mathrm{i})^{-1}=\mathrm{E}\left(\mathbf{x} \in \mathrm{C}_{\mathrm{a}, \mathrm{R}}\right)\left(\mathrm{H}_{\mathrm{o}}+1\right)^{-1} \cdot\left(\mathrm{H}_{\mathrm{o}}+1\right) \eta(\mathbf{Q})(\mathrm{H}+\mathrm{i})^{-1}$ is the product of a compact and a bounded operator, hence compact (cf. the proof of Lemma 2 ).

(ii) The proof of (H2) is almost identical with that of Lemma 3. By using (50) with $\Theta=\Sigma_{R}$, one obtains instead of (28) the inequality

$$
\left|\Phi_{\Sigma_{R}}\left(U_{t} g\right)\right| \leq 50\left\|\left(I+H_{0}\right) \eta(Q)(H+i)^{-1}\right\|^{2}\|(H+i) g\|^{2} .
$$

This is finite since $g \in D(H)$. In (29) $\theta_{R}(\mathbf{Q})$ is replaced by $\theta_{R}(\mathbf{Q}) \eta(\mathbf{Q})$, so that (30) must be applied to vectors of the form $\eta(Q) h$ with $h \in D(H)$ (by (44) such vectors belong to $D\left(H_{0}\right)$, hence also to $\left.\mathrm{D}\left(\mathrm{P}_{\mathrm{j}}\right)\right)$.

(iii) For $\theta=\Sigma_{a}$ or $\theta=\Lambda_{a, R}$ we write

$$
\Phi_{\Theta}\left(U_{t} g\right)=\Phi_{\Theta}\left(U_{t}^{1} f\right)+\left[\Phi_{\Theta}\left(U_{t} g\right)-\Phi_{\Theta}\left(U_{t}^{1} f\right)\right]
$$

We know from the proof of Lemma 4 (for $\theta=\Sigma_{\mathrm{a}}$ ) and from the proof of Proposition 3 (for $\theta$ $\left.=\Lambda_{a, R}\right)$ that $\left|\Phi_{\Theta}\left(U_{t}^{1} f\right)\right| \leq c t-\mu$ for some $\mu>1$ if $t \geq t_{o}>0$. Thus, to obtain (H3) and (H4) it suffices to show that $\left|\Phi_{\odot}\left(U_{t} g\right)-\Phi_{\odot}\left(U_{t}^{1} f\right)\right| \leq c t-\mu$ for some $\mu>1$, all $t \geq t_{0}$ and some constant $c$ independent of $\Theta$. To do this we write

$$
\begin{aligned}
& \delta \Phi_{\odot}(t) \equiv \Phi_{\Theta}\left(U_{t} g\right)-\Phi_{\Theta}\left(U_{t}^{1} f\right) \\
& =\operatorname{Re}\left[<(\mathrm{I}+\mathrm{P}) \eta(\mathbf{Q}) \mathrm{U}_{\mathrm{t}} \mathrm{g}, \mathrm{F}_{\mathrm{Q}}\left(\mathrm{I}+\mathrm{H}_{\mathrm{O}}\right) \eta(\mathbf{Q}) \mathrm{U}_{\mathrm{t}} \mathrm{g}>-<(\mathrm{I}+\mathrm{P}) \eta(\mathbf{Q})^{2} \mathrm{U}_{\mathrm{t}}^{1} \mathrm{f}, \mathrm{F}_{\mathrm{Q}}\left(\mathrm{I}+\mathrm{H}_{\mathrm{o}}\right) \eta(\mathbf{Q})^{2} \mathrm{U}_{\mathrm{t}}^{\mathrm{l}} \mathrm{f}>\right] \\
& =\operatorname{Re}\left[<(I+P) \eta(Q)\left(U_{t} g-\eta(Q) U_{t}^{1} f\right), F_{Q}\left(I+H_{0}\right) \eta(Q)^{2} U_{t}^{1} f>+\right. \\
& \left.+<(\mathrm{I}+\mathrm{P}) \eta(\mathbf{Q}) \mathrm{U}_{\mathrm{t}} \mathrm{g}, \mathrm{F}_{\Theta}\left(\mathrm{I}+\mathrm{H}_{\mathbf{0}}\right) \eta(\mathbf{Q})\left(\mathrm{U}_{\mathrm{t}} \mathrm{g}-\eta(\mathbf{Q}) \mathrm{U}_{\mathrm{t}}^{1} \mathrm{f}\right)>\right]
\end{aligned}
$$

It follows (with $\left.\left\|(I+P)\left(\mathrm{H}_{0}+\mathrm{I}\right)^{-1}\right\|<5 / 4\right)$ that

$$
\left|\delta \Phi_{\Theta}(t)\right| \leq \frac{5}{4}\left\|F_{\mathcal{Q}}\right\|\left[\left\|\left(I+H_{0}\right) \eta(Q)^{2} U_{t}^{1} f\right\|+\left\|(I+P) \eta(Q) U_{t} g\right\|\right]\left\|\left(I+H_{0}\right) \eta(Q)\left(U_{t} g-\eta(Q) U_{t}^{1} f\right)\right\| .
$$

We have $\left\|F_{\Sigma_{\mathrm{a}}}\right\|<\infty,\left\|\mathrm{F}_{\Lambda_{\mathrm{a}, \mathrm{R}}}\right\| \leq 3\left\|\Gamma_{\Lambda_{\mathrm{a}}}\right\|^{2}<\infty$ and 


$$
\begin{aligned}
& \left\|\left(\mathrm{I}+\mathrm{H}_{\mathrm{o}}\right) \eta(\mathbf{Q})^{2} \mathrm{U}_{\mathrm{t}}^{1} \mathrm{f}\right\| \leq\left\|\left(\mathrm{I}+\mathrm{H}_{\mathrm{O}}\right) \eta(\mathbf{Q})^{2}\left(\mathrm{H}_{1}+\mathrm{i}\right)^{-1}\right\|\left\|\left(\mathrm{H}_{1}+\mathrm{i}\right) \mathrm{f}\right\| \\
& \left\|(\mathrm{I}+\mathrm{P}) \eta(\mathrm{Q}) \mathrm{U}_{\mathrm{t}} \mathrm{g}\right\| \leq \frac{5}{4}\left\|\left(\mathrm{I}+\mathrm{H}_{\mathrm{o}}\right) \eta(\mathrm{Q})(\mathrm{H}+\mathrm{i})^{-1}\right\|\|(\mathrm{H}+\mathrm{i}) \mathrm{g}\| .
\end{aligned}
$$

Hence the inequality $\left|\delta \Phi_{\mathcal{O}}(t)\right| \leq \mathrm{ct}^{-\mu}$ follows as a consequence of Lemma 9 (in which we may choose $v^{\prime}>2$ ).

(iv) The last statement of the theorem now follows from Proposition 2 provided that (12) holds, i.e. provided that $\frac{d}{d t}\left\|E\left(x \in C_{a, R}\right) U_{t} g\right\|^{2}=-\Phi_{\partial C_{a, R}}\left(U_{t} g\right)$ with $\Phi_{\Theta}\left(U_{t} g\right)$ given by (50). If $V_{3}=0$ we know from the results of Section 3 (in particular (9), (11) and (13)) that this result holds, so the theorem is completely proven in this case.

If $V_{3} \neq 0$, the r.h.s. of (50) is finite (whereas the r.h.s. of (7), with $f=U_{t} g$, need not be defined because $\mathrm{U}_{\mathrm{t}} \mathrm{g}$ need not belong to $\mathrm{D}\left(\mathrm{H}_{\mathrm{O}}\right)$ ). One still has (see (11))

$$
\frac{d}{d t}\left\|E\left(x \in C_{a, t}\right) U_{t} g\right\|^{2}=i\left[<E\left(x \in C_{a, R}\right) H U_{t} g, U_{t} g>-<U_{t} g, E\left(x \in C_{a, R}\right) H U_{t} g>\right],
$$

since this result does not depend on the condition that $U_{t} g \in D\left(H_{0}\right)$. Now choose a real-valued function $\eta_{1}$ of class $C^{\infty}$ such that $\eta_{1}(\mathbf{x}) \eta(\mathbf{x})=\eta(\mathbf{x})$ for all $\mathbf{x} \in \mathbb{R}^{3}$ and $\eta_{1}(\mathbf{x})=0$ if $|\mathbf{x}|<\mathrm{r}_{\mathbf{0}}+$ $\left(a-r_{o}\right) / 10$, and let $h \in \mathcal{B}\left(\mathbb{R}^{3}\right)$. Observe that $P_{j}\left(\partial_{j} \eta\right)(Q) h=\eta_{1}(Q) P_{j}\left(\partial_{j} \eta\right)(Q) h$ and that $\eta(Q) U_{t} g$, $\eta_{1}(\mathbf{Q}) \mathrm{U}_{\mathrm{t}} \mathrm{g},\left(\partial_{\mathrm{j}} \eta\right)(\mathbf{Q}) \mathrm{U}_{\mathrm{t}} \mathrm{g}$ and $\eta(\mathbf{Q}) \mathrm{h}$ belong to $\mathrm{D}(\mathrm{H}) \cap \mathrm{D}\left(\mathrm{H}_{\mathrm{o}}\right) \cap \mathrm{D}(\mathrm{V})$ by (44). So

$$
\begin{aligned}
& \left\langle\eta(\mathbf{Q}) \mathrm{HU}_{\mathrm{t}} \mathrm{g}, \mathrm{h}\right\rangle=\left\langle\mathrm{U}_{\mathrm{t}} \mathrm{g}, \mathrm{H} \eta(\mathbf{Q}) \mathrm{h}\right\rangle=\left\langle\mathrm{U}_{\mathrm{t}} \mathrm{g}, \mathrm{H}_{\mathrm{o}} \eta(\mathbf{Q}) \mathrm{h}+\mathrm{V}(\mathbf{Q}) \eta(\mathbf{Q}) \mathrm{h}\right\rangle \\
& =\left\langle\mathrm{U}_{\mathrm{t}} \mathrm{g}, \eta(\mathbf{Q}) \mathrm{H}_{\mathrm{o}} \mathrm{h}+(\Delta \eta)(\mathbf{Q}) \mathrm{h}-2 \mathrm{iP} \cdot(\nabla \eta)(\mathbf{Q}) \mathrm{h}+\mathrm{V}(\mathbf{Q}) \eta(\mathbf{Q}) \mathrm{h}\right\rangle \\
& =\left\langle\mathrm{H}_{\mathrm{o}} \eta(\mathbf{Q}) \mathrm{U}_{\mathrm{t}} \mathrm{g}+(\Delta \eta)(\mathbf{Q}) \mathrm{U}_{\mathrm{t}} \mathrm{g}+2 \mathrm{i}(\nabla \eta)(\mathbf{Q}) \cdot \mathbf{P} \eta_{1}(\mathbf{Q}) \mathrm{U}_{\mathrm{t}} \mathrm{g}+\mathrm{V}(\mathbf{Q}) \eta(\mathbf{Q}) \mathrm{U}_{\mathrm{t}} \mathrm{g}, \mathrm{h}\right\rangle
\end{aligned}
$$

Hence

$$
\eta(\mathbf{Q}) \mathrm{HU}_{\mathrm{tg}} \mathrm{g}=\left[\mathrm{H}_{\mathrm{o}} \eta(\mathbf{Q})+(\Delta \eta)(\mathbf{Q})+2 \mathrm{i}(\nabla \eta)(\mathbf{Q}) \cdot \mathbf{P} \eta_{1}(\mathbf{Q})+\mathrm{V}(\mathbf{Q}) \eta(\mathbf{Q})\right] \mathrm{U}_{\mathrm{tg}}
$$

so that

$$
\mathrm{E}\left(\mathbf{x} \in \mathrm{C}_{\mathrm{a}, \mathrm{R}}\right) \mathrm{HU}_{\mathrm{t}} \mathrm{g}=\mathrm{E}\left(\mathbf{x} \in \mathrm{C}_{\mathrm{a}, \mathrm{R}}\right) \eta(\mathbf{Q}) \mathrm{HU}_{\mathrm{t}} \mathrm{g}=\mathrm{E}\left(\mathbf{x} \in \mathrm{C}_{\mathrm{a}, \mathrm{R}}\right) \mathrm{H}_{\mathrm{o}} \eta(\mathbf{Q}) \mathrm{U}_{\mathrm{t}} \mathrm{g}+\mathrm{V}(\mathbf{Q}) \mathrm{E}\left(\mathbf{x} \in \mathrm{C}_{\mathrm{a}, \mathrm{R}}\right) \mathrm{U}_{\mathrm{t}} \mathrm{g} .
$$

Substitution into (51) leads to

$$
\begin{aligned}
\frac{d}{d t} & \left\|E\left(x \in C_{a}, R\right) U_{t} g\right\|^{2} \\
& =i\left[<H_{0} \eta(Q) U_{t} g, E\left(x \in C_{a, R}\right) \eta(Q) U_{t} g>-<\eta(Q) U_{t} g, E\left(x \in C_{a, R}\right) H_{0} \eta(Q) U_{t} g>\right]
\end{aligned}
$$

An application of Lemma 1 confirms the validity of (12) with $\Phi_{\partial C_{\mathrm{a} R}}\left(\mathrm{U}_{\mathrm{t}} \mathrm{g}\right)$ as in (50). 
Remarks: (a) It is clear that the Coulomb potentials $\mathrm{V}(\mathbf{x})=\lambda|\mathbf{x}|^{-1}$ are covered by the theorem (take e.g. $V_{1}(x)=\eta(x) V(x)$ with $\eta$ as before, $V_{2}=V-V_{1}$ and $V_{3}=0$ ).

(b) The theorem gives the validity of (16) for a dense set of vectors $\mathrm{g}$ in the range $\mathrm{R}\left(\mathrm{W}_{+}\right)$of the relative wave operator $W_{+}$. In many situations, in particular if $V_{3}=0$, the operator $W_{+}$ maps $\mathfrak{H}_{\mathrm{ac}}\left(\mathrm{H}_{1}\right)$ onto $\mathfrak{H}_{\mathrm{ac}}(\mathrm{H})$ (see for example [19]-[21]), so that (16) holds for a dense set of vectors $g$ in $\mathcal{H}_{a c}(H)$. However, if $V_{3} \neq 0, R\left(W_{+}\right)$may be strictly smaller than $\mathcal{H}_{a c}(H)$. In this case one will have

$$
\mathcal{H}_{\mathrm{ac}}(\mathrm{H})=\mathbf{R}\left(\mathrm{W}_{+}\right) \oplus \mathfrak{m}_{\mathrm{g}}^{+}(\mathrm{H})
$$

(see [18] for the short range case). If $g \in R\left(W_{+}\right)$, then $\left\|E(|x| \leq a) U_{t} g\right\| \rightarrow 0$ as $t \rightarrow+\infty$, hence $\lim _{t \rightarrow+\infty}\left\|E(x \in C) U_{t}\right\|^{2}=\lim _{R \rightarrow \infty} \int_{T}^{\infty} \Phi_{\Sigma_{R}}\left(U_{t} g\right) d t$. The vectors $g$ in $\mathfrak{M}_{g}^{+}(H)$ are absorbed by the singularities of $V_{3}$ on $\mathcal{G}$ as $t \rightarrow+\infty$, in particular $\left\|E(|x| \geq a) U_{t} g\right\| \rightarrow 0$ as $t \rightarrow+\infty$ for $g \in \mathfrak{M}_{\rho}^{+}(H)$ [18]. So, if $g \in \mathcal{H}_{a c}(H)$ is of the form $g=W_{+} f \oplus g_{g}$ in the direct sum (52), with $f \in \mathscr{D}_{\rho}\left(H_{1}\right)$ $(\rho>2)$ and $g_{e} \in M_{e}^{+}(H)$, then

$$
\lim _{t \rightarrow+\infty}\left\|E\left(x \in C_{a}\right) U_{t} g\right\|^{2}=\lim _{t \rightarrow+\infty}\left\|E\left(x \in C_{a}\right) U_{t} W_{+} f\right\|^{2}=\lim _{R \rightarrow \infty} \int_{T}^{\infty} \Phi_{\Sigma_{R}}\left(U_{t} W_{+} f\right) d t
$$

(c) Our results can be related to scattering theory. Consider the wave operators $\Omega_{ \pm}=$

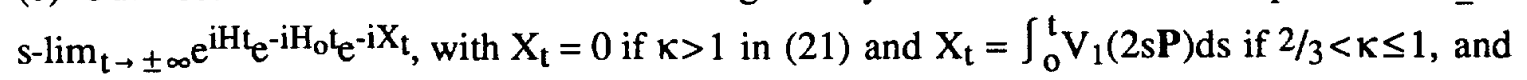
let $S=\Omega_{+}^{*} \Omega_{-}$be the associated scattering operator. If $f_{\text {in }}$ is an initial state for scattering by the potential $\mathrm{V}$, then

$$
\lim _{t \rightarrow+\infty}\left\|E\left(x \in C_{a}\right) U_{t} \Omega_{-} f_{i n}\right\|^{2}=\int_{C}\left|\left(\mathcal{F S f} f_{\text {in }}\right)(k)\right|^{2} d^{3} k
$$

(see e.g. [22], Lemma 6.6 of [1] or Chapter 9.2 of [2]; $T h$ denotes the Fourier transform of h).

(d) Our results cover scattering by obstacles in the following sense. If $\zeta$ is the surface of a simply connected bounded set $G$ in $\mathbb{R}^{3}$, for example a sphere, then there are self-adjoint extensions $\mathrm{H}$ of $\hat{H}$ that are decoupled by $\mathcal{G}$ [23], i.e. $\mathrm{H}=\mathrm{H}_{\mathrm{i}} \oplus \mathrm{H}_{\mathrm{e}}$ in the representation $L^{2}(G) \oplus L^{2}\left(\mathbb{R}^{3} \backslash G\right)$ of $\mathcal{H}$, and one may apply our theorem to vectors in $\boldsymbol{H}_{a c}\left(H_{e}\right)$.

Acknowledgments : This work was carried out during the autumn 1996 when both authors were at CERN. We wish to express our thanks to the Theory Division of CERN for its kind hospitality. 


\section{Appendix}

\section{Proof of Proposition 1 :}

(i) We embed $\theta$ in a shell $G_{\delta}$ of thickness $2 \delta>0$. To do this, let $\theta_{\lambda}$ be the surface obtained from $\Theta$ by a displacement of length $\lambda$ orthogonal to $\Theta$, i.e. $\Theta_{\lambda}=\left\{\mathbf{x} \in \mathbb{R}^{3} \mid \mathbf{x}=\mathbf{y}+\right.$ $\lambda \mathbf{n}(\mathbf{y}), \mathbf{y} \in \mathcal{\Theta}$, and let $\mathrm{G}_{\delta}=U_{-\delta \leq \lambda \leq \delta} \Theta_{\lambda}$. If $\Theta=\Sigma_{\mathrm{b}}$, we take $\delta=b / 2$; if $\Theta=\Theta_{k}$, we choose $\delta$ sufficiently small so that each vector $x$ in $G_{\delta}$ has a unique decomposition into $x=y+\lambda \mathbf{n}(\mathbf{y})$ with $\mathbf{y} \in \mathcal{O}, \lambda \in[-\delta, \delta]$. Throughout this proof it is understood that $\mathbf{x}$ and $\mathbf{y}$ are related in the preceding manner (so, as $\mathbf{x}$ varies over $\mathrm{G}_{\delta}, \mathbf{y}$ and $\lambda$ are functions of $\mathbf{x}$ ).

If $d \sigma_{\lambda}(\mathbf{y})$ denotes the surface element on $\Theta_{\lambda}$ at the point $\mathbf{y}+\lambda \mathbf{n}(\mathbf{y})$ and $d \sigma(\mathbf{y}) \equiv$ $\mathrm{d} \sigma_{\mathrm{o}}(\mathrm{y})$ the corresponding quantity on $\Theta$, then there is a constant $\gamma \in[1, \infty)$ such that

$$
\frac{1}{\gamma} \mathrm{d} \sigma(\mathbf{y}) \leq \mathrm{d} \sigma_{\lambda}(\mathbf{y}) \leq \gamma \mathrm{d} \sigma(\mathbf{y}) \quad \forall \mathbf{y} \in \Theta, \forall \lambda \in[-\delta, \delta]
$$

(ii) Let $f$ be as stated in the proposition and $x \in G_{\delta}$. For $\rho \in[0,1]$, set

$$
h_{\rho}^{(j)}(x)=\left(\partial_{j} f\right)(y+\rho(x-y))=i\left(P_{j} f\right)(y+\rho \lambda \mathbf{n}(\mathbf{y}))
$$

By Newton's formula one has

$$
f(x)=f(y)+\Sigma_{j=1}^{3}\left(x_{j}-y_{j}\right) \int_{o}^{1} h_{\rho}^{(j)}(x) d \rho .
$$

This implies that

$$
\begin{aligned}
& \int_{\Theta}|f(y)|^{2} \mathrm{~d} \sigma(\mathbf{y})=\frac{1}{2 \delta} \int_{-\delta}^{\delta} \mathrm{d} \lambda \int_{\Theta}|\mathrm{f}(\mathbf{y})|^{2} \mathrm{~d} \sigma(\mathbf{y}) \leq \frac{\gamma}{2 \delta} \int_{-\delta}^{\delta} \mathrm{d} \lambda \int_{\mathcal{Q}}|\mathrm{f}(\mathbf{y})|^{2} \mathrm{~d} \sigma_{\lambda}(\mathbf{y}) \\
& \leq \frac{2 \gamma}{2 \delta} \int_{\mathrm{G}_{\delta}}|\mathrm{f}(\mathbf{x})|^{2} \mathrm{~d}^{3} \mathbf{x}+\frac{2 \gamma}{2 \delta} \delta^{2} \Sigma_{\mathrm{j}=1}^{3} \int_{\mathrm{G}_{\delta}} \mathrm{d}^{3} \mathbf{x}\left|\int_{\mathrm{o}}^{1} h_{\rho}^{(j)}(\mathbf{x}) \mathrm{d} \rho\right|^{2} \\
& \leq \frac{\gamma}{\delta}\|f\|^{2}+\delta \gamma \Sigma_{j=1}^{3}\left\|\int_{\mathrm{o}}^{1} h_{\rho}^{(j)} \mathrm{d} \rho\right\|_{L^{2}\left(G_{\delta}\right)}^{2} .
\end{aligned}
$$

Now by the triangle inequality

$$
\left\|\int_{0}^{1} h_{\rho}^{(j)} d \rho\right\|_{L^{2}\left(G_{\delta}\right)} \leq \int_{0}^{1}\left\|h_{\rho}^{(j)}\right\|_{L^{2}\left(G_{\delta}\right)} d \rho,
$$

where (for $0<\rho \leq 1$, and by using (53)): 


$$
\begin{aligned}
& \left\|h_{\rho}^{(j)}\right\|_{L}^{2}\left(G_{\delta}\right)=\int_{-\delta}^{\delta} d \lambda \int_{\Theta} d \sigma_{\lambda}(y)\left|h_{p}^{(j)}(y+\lambda n(y))\right|^{2}=\int_{-\delta}^{\delta} d \lambda \int_{\Theta} d \sigma_{\lambda}(y)\left|\left(P_{j} f\right)(y+\rho \lambda n(y))\right|^{2} \\
& =\frac{1}{\rho} \int_{-\rho \delta}^{\rho \delta} \mathrm{d} \mu \int_{\Theta} \mathrm{d} \sigma_{\mu / \rho}(\mathbf{y})\left|\left(\mathrm{P}_{\mathrm{j}} \mathrm{f}\right)(\mathbf{y}+\mu \mathbf{n}(\mathbf{y}))\right|^{2} \leq \frac{\gamma^{2}}{\rho} \int_{-\delta}^{\delta} \mathrm{d} \mu \int_{\Theta} \mathrm{d} \sigma_{\mu}(\mathbf{y})\left|\left(P_{\mathrm{j}} f\right)(\mathbf{y}+\mu \mathbf{n}(\mathbf{y}))\right|^{2} \\
& =\frac{\gamma^{2}}{\rho} \| \mathrm{P}_{\mathrm{j}} \mathrm{fl} \mathrm{L}^{2}\left(\mathrm{G}_{\delta}\right)
\end{aligned}
$$

Together with (56) and (57), this implies that

$$
\|f\|_{\theta}\left\|_{L}^{2}(\theta) \leq \frac{\gamma}{\delta}\right\| f\left\|^{2}+\delta \gamma^{3}\left[\int_{o}^{1} \rho^{-1 / 2} d \rho\right]^{2} \Sigma_{j=1}^{3}\right\| P_{j} f \|^{2}
$$

So (2) holds with $\varphi \equiv 1$, with $c=\sqrt{\gamma} \cdot \max \left(\delta^{-1 / 2}, 2 \gamma \sqrt{\delta}\right)$. The result for general $\varphi$ follows immediately because $\mathrm{f} l_{\Theta}=\left.[\varphi \mathrm{f}]\right|_{\Theta}$.

\section{Proof of the estimate (5):}

Take $\mathcal{O}=\Sigma_{\mathrm{b}}$ in Proposition 1 , and let $\mathrm{c}_{\mathrm{b}}$ be a constant such that (2) holds with $\mathrm{c}=\mathrm{c}_{\mathrm{b}}$ in this case. We have seen after Proposition 1 that $\left\|\Gamma_{\Sigma_{b}}\right\| \leq \sqrt{2} c_{b}$, and from the above proof of Proposition 1 we know that $c_{b} \leq \sqrt{\gamma_{b}} \cdot \max \left(\delta^{-1 / 2}, 2 \gamma_{b} \sqrt{\delta}\right)$, where $\gamma_{b} \geq 1$ is such that

$$
\frac{1}{\gamma_{b}} b^{2} \leq(b+\lambda)^{2} \leq \gamma_{b} b^{2} \quad \forall \lambda \in[-\delta, \delta]
$$

(because $d \sigma_{\lambda}(y)=(b+\lambda)^{2} \sin \theta d \theta d \varphi$ in spherical polar coordinates). Since we consider only values of $b$ that are $\geq 1$, we may chose $\delta=1 / 4$ (rather than $\delta=b / 2$ ) in the proof of Proposition 1. Then (60) is satisfied for all $b \geq 1$ and all $\lambda \in[-1 / 4,1 / 4]$ with $\gamma_{b}=2$. Since this number is independent of $b$, we get a bound on $\left\|\Gamma_{\Sigma_{b}}\right\|$ that does not depend on $b$ (explicitly $\left\|\Gamma_{\Sigma_{b}}\right\| \leq 4$; the estimate is not optimal). 


\section{References}

[1] Amrein W O 1981 Non-Relativistic Quantum Dynamics (Dordrecht: Reidel)

[2] Pearson D B 1988 Quantum Scattering and Spectral Theory (London: Academic Press)

[3] Combes J M, Newton R G and Shtokhamer R 1975 Phys. Rev. D 11366

[4] Daumer M, Dürr D, Goldstein S and Zanghi M 1996 Lett. Math. Phys. 38103

[5] Daumer M 1995 Streutheorie aus der Sicht Bohmscher Mechanik (Aachen: Verlag Mainz)

[6] Amrein W O and Zuleta J L 1997 Helv. Phys. Acta (to appear)

[7] Jeffreys H and Jeffreys B 1972 Methods of Mathematical Physics (Cambridge: University Press)

[8] Courant R 1936 Differential and Integral Calculus II (New York: Interscience)

[9] Adams R A 1975 Sobolev Spaces (New York: Academic Press)

[10] Reed M and Simon B 1975 Methods of Modern Mathematical Physics II (New York: Academic Press)

[11] Yajima K 1995 J. Math. Soc. Japan 47551

[12] Ikebe T and Saito Y 1972 J. Math. Soc. Kyoto Univ. 12513

[13] Amrein W O, Cibils M B and Sinha K B 1987 Ann. Inst. Henri Poincaré 47367

[14] Jensen A, Mourre E and Perry P 1984 Ann. Inst. Henri Poincaré 41207

[15] Jensen A 1985 Trans. Amer. Math. Soc. 291129

[16] Isozaki H 1986 J. Math. Soc. Kyoto Univ. 26595

[17] Radin C and Simon B 1978 J. Differential Equations 29289

[18] Pearson D B 1975 Helv. Phys. Acta 48639

[19] Lavine R 1973 J. Funct. Analysis 1230

[20] Pearson D B 1974 Helv. Phys. Acta 47249

[21] Amrein W O and Georgescu V 1974 Helv. Phys. Acta 47517

[22] Dollard J D 1969 Comm. Math. Phys. 12193

[23] Deift P and Simon B 1976 J. Funct. Analysis 23218 